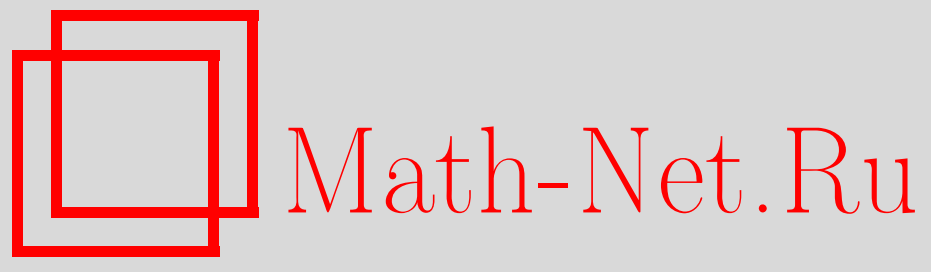

П. Я. Грозман, Д. А. Лейтес, Неголономные тензоры Римана и Вейля для флаговых многообразий, ТМФ, 2007, том 153, номер 2, 186-219

DOI: https://doi.org/10.4213/tmf6135

Использование Общероссийского математического портала Math-Net.Ru подразумевает, что вы прочитали и согласны с пользовательским соглашением http://www . mathnet.ru/rus/agreement

Параметры загрузки:

IP : 54.157 .27 .8

26 апреля 2023 г., 14:48:40

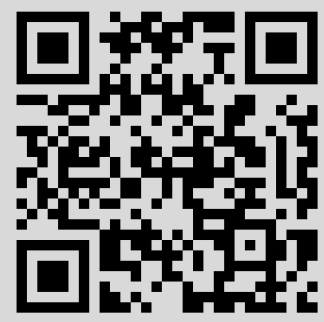




\title{
НЕГОЛОНОМНЫЕ ТЕНЗОРЫ РИМАНА И ВЕЙЛЯ ДЛЯ ФЛАГОВЫХ МНОГООБРАЗИЙ
}

\begin{abstract}
На любом многообразии любая невырожденная симметрическая 2-форма (метрика) и любая невырожденная кососимметрическая дифференциальная форма $\omega$ могут быть приведены к каноническому виду в любой точке, но не в каждой окрестности: соответствующими препятствиями являются тензор Римана и $d \omega$. Препятствия к плоскостности (приводимости к каноническому виду) хорошо известны для любой $G$-структуры, а не только для римановой и симплектической структур. Для многообразий с неголономной структурой (с неинтегрируемым распределением) общие определения плоскостности и препятствий к ней, представляющие огромный интерес (например, в супергравитации), не были известны до недавнего времени, хотя их частные случаи известны более столетия (например, любая контактная структура неголономно плоская: локально ее всегда можно привести к каноническому виду).

Дано общее определение неголономных аналогов тензора Римана и его конформно инвариантного аналога - тензора Вейля - в терминах когомологий алгебр Ли, а также изложны теоремы Премета, описывающие эти когомологии. С помощью теорем Премета и пакета программ SuperLie вычислены тензоры для флаговых многообразий, ассоциированных с каждой максимальной параболической подалгеброй каждой простой алгебры Ли (и еще в нескольких случаях), а также препятствия к плоскостности $G(2)$-структуры и ее неголономного супераналога.
\end{abstract}

Ключевые слова: когомологии алгебр Ли, картановские продолжения, тензор Римана, неголономное многообразие, многообразие флагов, $G(2)$-структура.

\section{1. ВВЕДЕНИЕ}

Герц [1] ввел термин неголономная динамическая система, пытаясь геометрически описать движение так, чтобы изгнать из математического и физического описаний движений понятие силы. Многообразие (фазовое пространство динамической

*Equa Simulation AB, Stockholm, Sweden. E-mail: pavel@rixetele.com

${ }^{\dagger}$ Max-Planck-Institut für Mathematik in der Naturwissenschaften, Leipzig, Germany; Department of Mathematics, University of Stockholm, Stockholm, Sweden.

E-mail: mleites@math.su.se 
системы) называется неголономным, если оно наделено неинтегрируемым распределением, т.е. неинтегрируемым подрасслоением касательного расслоения. Простейшим механическим примером неголономной динамической системы является твердое тело, катящееся без скольжения по другому телу. Среди разнообразных картин, которые приходят на ум, самой наивной является шар на шершавой плоскости [2] или велосипед, катящийся по асфальту. В точке касания колеса с асфальтом скорость колеса равна нулю. (Это линейная связь. Мы не будем здесь рассматривать более общие нелинейные связи, хотя они тоже естественны: в любом движущемся автомобиле, снабженном контролем скорости, включите этот контроль.) Известная теорема Фробениуса дает критерий локальной интегрируемости распределений: сечения распределения должны образовывать алгебру Ли.

Исторический обзор неголономных систем имеется в [3], а очень интересная работа Вершика [4] дает первое строгое математическое описание неголономной геометрии и указывает на возможные применения в разнообразных областях, часто неожиданных для того времени, когда эта работа была сделана (например, в задачах оптимального управления или в макроэкономике, где нелинейные связи тоже вполне естественны, см. [5]-[7]). Очень полезна также недавняя книга Козлова [8]. В работе [4] Вершик суммирует результаты примерно ста лет изучения неголономной геометрии (Герц, Каратеодори, Врэнчану, Вагнер, Схоутен, Фаддеев, Гриффитс, Годбийон); a MathSciNet показывает тысячи статей со словом "неголономный" и его синонимами (анголономный, субриманов, автопараллельный) и частными случаями, приводящими к неголономным связям (финслеров случай, “задача кошки”). Похоже, что неголономных систем в каком-то смысле даже "больше", чем голономных.

Сравнительно новая теория - супергравитация (теория, овеществляющая мечту Эйнштейна об объединенной теории поля) - тоже имеет дело исключительно с неголономными структурами, но не на многообразиях, а на супермногообразиях.

В начале XX века Каратеодори показал, что термодинамика является неголономной системой, а в 1990-х годах Сергеев показал, что рыночную экономику можно рассматривать как вариант термодинамики, следовательно, тоже как неголономную систему [7], [9].

В конце статьи [4] Вершик резюмировал не удавшиеся за столетие попытки исследователей дать определения аналогу тензора Римана для общего неголономного многообразия, предположив, что "хотя он и известен в некоторых случаях, его, вероятно, невозможно определить в общем случае". Однако в 1989 г. один из авторов настоящей статьи (Д.Л.), будучи в Институте высших исследований (Принстон), дал такое общее определение и в дальнейшем делал доклады о нем на различных школах и конференциях (в Международном центре теоретической физики (Триест, Италия), Международном математическом институте им. Л. Эйлера (Санкт-Петербург), ОИЯИ и т.д.), см. [10], [11]. Позже мы применили это определение к супергравитации [12]. Недавно стали появляться и переложения работ [10], [12], причем без ссылок. 
Оказывается, что примерно десятью годами раньше Лейтеса Танака [13]-[15] дал, в сущности, такое же определение "неголономной версии тензора Римана". Он рассматривал свою задачу по совершенно другим причинам (в работах Танаки и даже в недавних обзорных статьях [16], [17] слово “неголономный” ни разу даже не упоминается). Результаты Танаки (и особенно их ясное изложение в статье Ямагучи [16]) легче понять, чем первые попытки, принадлежащие Схоутену и Вагнеру, см. [18], поскольку после нескольких экспериментов Танака использовал иероглифику когомологий алгебр Ли, которая гораздо более наглядна, чем координатные тензорные обозначения. Тензор Танаки совпадает с тем, что мы предложили для нужд супергравитации в [10], если ограничиться несуперслучаем.

Всюду в дальнейшем основное поле - это поле $\mathbb{C}$ комплексных чисел.

Основными результатами этой статьи являются следующие:

1) пояснение общего определения неголономного аналога тензора Римана и его конформного (вейлевского) аналога;

2) теоремы Премета, которые облегчают вычисления этих тензоров в некоторых случаях (для флаговых многообразий $G / P$, где $G$ - простая группа Ли, а $P$ - ее параболическая подгруппа);

3) вычисление неголономных аналогов тензоров Римана и Вейля в нескольких частных случаях (в случаях простейших аналогов “классических областей”); при этом мы используем теоремы Премета и основанный на пакете Mathematica пакет SuperLie [19].

Вычисления неголономных аналогов тензора Римана довольно трудны с технической точки зрения. Редкими исключениями среди работ, посвященных таким аналогам, с действительно вычисленными результатами, являются работы [16], [17], [20]-[29], в которых в несуперпостановке использовано тождественное нашему определение Танаки неголономного тензора Римана. Однако авторы этих работ не были вооружены теоремами Премета и пакетом SuperLie и поэтому не могли получить те результаты, которые в настоящее время может получить каждый с помощью этих орудий и алгоритма Щепочкиной $[30]^{1)}$.

1.1. Общее описание классических тензоров. В середине 1970-х годов Гиндикин сформулировал проблему локальной характеризации "классических областей" - компактных эрмитовых симметрических областей $X=S / P$, где $S$ - простая группа Ли, а $P$ - ее параболическая подгруппа. Гончаров решил эту проблему [32], рассмотрев поля некоторых квадратичных конусов и вычислив структурные функции (препятствия к плоскостности) соответствующих $G$-структур, где $G$ - (редуктивная) подгруппа Леви группы $P$.

1) В 2000 г. Вакару сообщил нам о своих результатах и результатах математиков школы Вранчану, частично суммированных в книге [31] и многочисленных приведенных в ней ссылках. Через лес неинвариантных сложных тензорных выражений непросто разглядеть, что в [31], также как и в [18], отсутствуют некоторые компоненты по сравнению с определениями Танаки и нашим. 
1.1.1. Примеры. При $S=O(n+2)$ и $G=C O(n)=O(n) \times \mathbb{C}^{\times}$структурные функции известны (здесь $\left.\mathbb{C}^{\times}=\mathbb{C} \backslash\{0\}\right)$. Они составляют тензор Вейля - конформноинвариантную часть риманова тензора кривизны.

При $S=S L(n+m)$ и $G=S(G L(n) \times G L(m))$ структурные функции являются препятствиями к интегрируемости многомерных аналогов $\alpha$ - и $\beta$-плоскостей Пенроуза на грассманиане $\mathrm{Gr}_{n}^{n+m}$ (Пенроуз рассматривал $\mathrm{Gr}_{2}^{4}$ ).

Не для каждой простой группы Ли $S$ и ее подгруппы $P$ можно образовать классическую область: $S$ не может быть изоморфна $G(2)^{2)}, F(4)$ и $E(8)$, а $P$ может быть лишь максимальной параболической подгруппой $P_{i}$, порожденной всеми образующими Шевалле группы $S$ за исключением одного $(i$-го), скажем, отрицательного. Группа $P$ или, что то же самое, $i$-я образующая Шевалле группы $S$, которую в дальнейшем мы будем называть избранной, тоже не может быть произвольной. Чтобы описать допустимые подгруппы $P$, пометим вершины графа Дынкина группы $S$ коэффициентами линейной зависимости максимального корня относительно простых корней. Избранная образующая может отвечать только вершине с меткой 1.

Для каждой простой группы Ли $S$ зафиксируем произвольную $\mathbb{Z}$-градуировку ее алгебры Ли $\mathfrak{s}=\operatorname{Lie}(S)$. Для любой подгруппы $P \subset S$, порожденной неотрицательными элементами алгебры Ли $\mathfrak{s}$, естественно поставить следующие вопросы:

каков аналог конформной структуры Гончарова;

каковы соответствующие аналоги проективных структур

и римановых структур;

какие из этих структур плоские в каком-то неголономном смысле;

каковы препятствия к их плоскостности?

1.1.2. Замечание. Прилагательное "произвольная" в описании $\mathbb{Z}$-градуировки алгебры э появилось благодаря И. Бернштейну. Все $\mathbb{Z}$-градуировки получаются, если для образующих Шевалле $X_{i}^{ \pm}$положить $\operatorname{deg} X_{i}^{ \pm}= \pm k_{i}$, где $k_{i} \in \mathbb{Z}$; мы рассматриваем случай, когда $k_{i} \geqslant 0$ при всех $i$. Недавно Костант [33] рассмотрел аналог теоремы Ботта-Бореля-Вейля (ББВ) - один из наших основных инструментов - для всех $\mathbb{Z}$-градуировок, но его ответ недостаточно алгебраичен для нас и SuperLie.

Современное описание структурных функций обычно дается в терминах когомологий Спенсера, см. [34], [35] (ниже мы напомним все определения нужные для понимания задач (1) и формулы (2)). Гончаров записал структурные функции как тензоры, принимающие значение в векторном расслоении над $X=G / P$, слои которого в каждой точке $x \in X$ изоморфны друг другу и пространству

$$
H^{2}\left(\mathfrak{g}_{-1} ;\left(\mathfrak{g}_{-1}, \mathfrak{g}_{0}\right)_{*}\right), \quad \mathfrak{g}_{0}=\operatorname{Lie}(G), \quad \mathfrak{g}_{-1}=T_{x} X,
$$

где $\left(\mathfrak{g}_{-1}, \mathfrak{g}_{0}\right)_{*}=\bigoplus_{i \geqslant-1} \mathfrak{g}_{i}$ является картановским продолжением пары $\left(\mathfrak{g}_{-1}, \mathfrak{g}_{0}\right)$, см. далее формулу (3). Привычное представление структурных функций в терминах

2) Мы обозначаем исключительные группы и их алгебры Ли так же, как серийные, например, $S L(n)$, чтобы не путать $\mathfrak{g}(2)$ с $\mathfrak{g}_{2}-$ компонентой второй степени $\mathbb{Z}$-градуированной алгебры Ли $\mathfrak{g}$. 
биградуированных когомологий Спенсера $H^{k, 2}$ можно восстановить в любой момент, взяв в $H^{2}\left(\mathfrak{g}_{-1} ;\left(\mathfrak{g}_{-1}, \mathfrak{g}_{0}\right)_{*}\right)$ однородную компоненту степени $k$, отвечающую $\mathbb{Z}$-градуировке алгебры Ли $\left(\mathfrak{g}_{-1}, \mathfrak{g}_{0}\right)_{*}$.

1.2. Неголономные тензоры и наши примеры. Примерно в то же время, когда Гончаров получил свой результат, физики пытались написать уравнение супергравитации (для стандартных или “экзотических" $N$-супергравитаций), см. [26], [27], [36], [37] и столкнулись с теми же вопросами (1), но не для группы $S$, а для супергруппы $S=S L(4 \mid N)$ при $N \leqslant 8$ и подсупергруппы $P$, порожденной всеми аналогами образующих Шевалле супергруппы $G$, кроме двух. Соответствующее суперпространство $X=S / P$ является суперпространством флагов, а трудности с супергравитацией, упомянутые, например, в [36] (“мы не знаем, как определить аналог тензора Римана при $N>1$ "3), другими словами, "мы не знаем, что должно стоять в левой части уравнений супергравитации" при $N>1$, говорит Весс), были вызваны не суперприродой супермногообразия $X$, а его неголономностью.

Щепочкина ввела неголономные обобщения $\left(\mathfrak{g}_{-}, \mathfrak{g}_{0}\right)_{*}$ картановского продолжения $\left(\mathfrak{g}_{-1}, \mathfrak{g}_{0}\right)_{*}$ для классификации простых бесконечномерных супералгебр Ли векторных полей [38]. Она переоткрыла и суперизовала обобщение картановского продолжения Танаки и ввела несколько новых типов продолжений, например, частичное продолжение [39]-[41]. Эти продолжения, первоначально введенные для того, чтобы определить новые простые супералгебры векторных полей над $\mathbb{C}$ и $\mathbb{R}$, а недавно востребованные для интерпретации известных простых алгебр Ли над полями характеристики $p>0$ и обнаружения новых, являются в точности тем, что нам необходимо для определения неголономного аналога тензоров Вейля и Римана в общем случае.

Заметим, что наши неголономные инварианты, хотя и являются естественными аналогами тензоров кривизны и кручения, не совпадают на неголономных многообразиях с классическими тензорами, носящими те же имена. Например, на любом неголономном многообразии классическое кручение отлично от нуля по определению неголономности (форма Фробениуса, которая паре сечений распределения сопоставляет их скобку, не равна тождественно нулю), в то время как, например, каждое контактное многообразие плоско в нашем неголономном смысле. Чтобы избежать недоразумений, мы всегда должны добавлять прилагательное “неголономный” для инвариантов, которые мы введем ниже. Для краткости мы будем говорить о неголономном тензоре кривизны и уточнять при необходимости его степень (порядок структурной функции). Наложение связей Весса-Зумино [36] означает требование равенства нулю структурных функций меньших степеней, необходимое для определения структурных функций рассматриваемой степени.

Главная задача - ответить на вопросы (1). Сделав это (дав соответствующие определения в общем случае многообразия с неголономной структурой), мы для иллюстрации явно вычислим аналоги тензоров (2) - пространства неголономных структурных функций или возможных значений неголономных версий тензоров Вейля

\footnotetext{
3) Решение при $N \leqslant 3$ было найдено группой Огиевецкого, см. [26].
} 
и Римана. Мы проделаем это для простейших неголономных флаговых многообразий вида $S / P$ с одной избранной образующей Шевалле. В большинстве наших примеров продолжение $\left(\mathfrak{g}_{-}, \mathfrak{g}_{0}\right)_{*}$ совпадает с алгеброй Ли $\mathfrak{s}$ группы $S$, и поэтому можно применить теорему ББВ, воспроизведенную ниже (подробности см. в замечательном обзоре [42]). Если $\left(\mathfrak{g}_{-}, \mathfrak{g}_{0}\right)_{*}$ строго содержит $\mathfrak{s}$, то мы рассматриваем значения коциклов и в $\mathfrak{s}$, и в $\left(\mathfrak{g}_{-}, \mathfrak{g}_{0}\right)_{*}$.

Мы приводим теоремы Премета, которые показывают, как вычислить неголономные тензоры Вейля и Римана в общем случае, и используем эти теоремы, чтобы получить явный ответ в конкретных случаях.

Неявный вид ответа в [32] скрыл симметрию, описанную в работе [43], где, используя явный вид ответа, мы предложили аналоги уравнений Эйнштейна для некоторых специальных грассманианов. Среди случаев, которые мы здесь рассматриваем, феномен, похожий на описанный в [43], имеет место, например, для вершин при основании вилок в схемах Дынкина алгебр Ли $\mathfrak{e}(6)$ и o(8). Мы надеемся рассмотреть соответствующие аналоги уравнений Эйнштейна в другой статье.

Демонстрируя пользу пакета SuperLie, мы вычисляем также структурные функции $G(2)$-структуры, столь популярной в последнее время (см. [44]-[46]). Пакет SuperLie уже доказал свою полезность во многих случаях (ср. усилия для получения результатов статьи [47] с мучительным аналитическим счетом в [48] и [49]). Он совершенно необходим при изучении супералгебр Ли: практически ни для одной из них не существует ничего столь же компактно формулируемого, как теорема ББВ [50]. Мы также применяем пакет SuperLie для того, чтобы вычислить структурные функции одной из суперверсий $G(2)$-структур на проективном суперпространстве $\mathbb{C} P^{1,7}$ с неголономным распределением.

1.3. Замечание (связь с дифференциальными уравнениями). Пусть $\mathfrak{l}=$ $\mathfrak{l}_{-1} \oplus \mathfrak{l}_{0} \oplus \mathfrak{l}_{1}-$ конечномерная простая градуированная алгебра Ли над $\mathbb{C}$, а $M-$ конечномерный точный неприводимый l-модуль. Тогда $M=\bigoplus_{p \leqslant-1} M_{p}$, где

$$
M_{-1}=\left\{s \in M \mid \mathfrak{l}_{1} s=0\right\}, \quad M_{p}=\left(\mathfrak{l}_{-1}\right)^{-p-1} M_{-1} \quad \text { при } \quad p<0 .
$$

В [17] Ямагучи и Яцуи рассмотрели полупрямую сумму $\mathfrak{g}=M \in \mathfrak{l}$, где $[M, M]=0$, с естественной градуировкой. Они доказали, что $\mathfrak{m}=\bigoplus_{p<0} \mathfrak{g}_{p}$ есть алгебра символов некоторого дифференциального уравнения конечного типа. Модельное уравнение такого типа было построено Се-Аши [51].

При каких условиях алгебра Ли $\mathfrak{g}$ является продолжением алгебры Ли м или пары $\left(\mathfrak{m}, \mathfrak{g}_{0}\right)$ ? В качестве фундаментального инварианта Ямагучи и Яцуи предложили обобщенные когомологии Спенсера $H^{*}:=H^{*}(\mathfrak{m} ; \mathfrak{g})$ или $H^{*}\left(\mathfrak{m} \oplus \mathfrak{g}_{0} ; \mathfrak{g}\right)$ в наших обозначениях. Используя теорему Костанта, Ямагучи и Яцуи вычислили $H^{i}$ при $i=1,2$. Вычисление пространства $H^{1}$ дает полный ответ на поставленный выше вопрос, а явные вычисления пространства $H^{2}$ показывают, что за исключением трех случаев системы дифференциальных уравнений типа $\mathfrak{m}$ локально изоморфны модельной системе типа $(\mathfrak{l}, M)$.

Геометрическая интерпретация пространства $H^{2}(\mathfrak{m} ; \mathfrak{g})$ как препятствия к существованию нормальной связности Картана описана в [52]. 


\section{2. СТРУКТУРНЫЕ ФУНКЦИИ G-СТРУКТУР}

Пусть $M^{n}$ - многообразие над полем $\mathbb{C}$. Пусть $F M$ - расслоение реперов над $M$, т.е. главное $G L(n)$-расслоение. Пусть $G \subset G L(n)$ - группа Ли. $G$-структурой на $M$ называется редукция главного $G L(n)$-расслоения к главному $G$-расслоению. Есть и более вразумительная эквивалентная формулировка: $G$-структура - это такой выбор функций перехода от одной координатной окрестности к другой, что функции перехода принадлежат группе $G$ для каждой пары пересекающихся окрестностей.

Итак, в определении $G$-структуры участвуют следующие персонажи: многообразие $M^{n}$, два векторных расслоения $T M$ и $F M$ на нем и две группы $G$ и $G L(n)$, $G \subset G L(n)$, каждая из которых действует в каждом слое каждого расслоения.

Простейшая $G$-структура - это плоская $G$-структура, определенная следующим образом. В качестве модельного многообразия с плоской $G$-структурой возьмем $V=\mathbb{C}^{n}$ с фиксированным репером. Ключевой момент наших построений - отождествление касательных пространств $T_{v} V$ в разных точках $v$, что достигается с помощью параллельных переносов вдоль вектора $v$. Это означает, что мы рассматриваем пространство $V$ как коммутативную группу Ли и отождествляем пространства, касательные к ней в разных точках, с ее алгеброй Ли vo. В силу коммутативности $\mathfrak{v}$ можно естественным образом отождествить с самим пространством $V$, при этом неважно, используем ли мы левые или правые сдвиги.

Таким образом, мы получаем фиксированный репер в каждом пространстве $T_{v} V$. Плоская $G$-структура - это расслоение над $V$, в котором слой над точкой $v \in V$ состоит из всех реперов, полученных из данного репера с помощью $G$-действия. В учебниках по дифференциальной геометрии, например, в [34], препятствия к отождествлению $k$-й инфинитезимальной окрестности точки $m \in M$ (на многообразии $M$ с $G$-структурой) с $k$-й инфинитезимальной окрестностью точки на многообразии $V$ с описанной выше плоской $G$-структурой называются структурными функииями порядка $k$.

Чтобы точно описать структурные функции, положим

$$
\mathfrak{g}_{-1}=T_{m} M, \quad \mathfrak{g}_{0}=\mathfrak{g}=\operatorname{Lie}(G)
$$

Напомним, что для любого (конечномерного) векторного пространства $V$ имеет место изоморфизм

$$
\operatorname{Hom}(V, \operatorname{Hom}(V, \ldots, \operatorname{Hom}(V, V) \ldots)) \simeq L^{i}(V, V, \ldots, V ; V)
$$

где $L^{i}$ - пространство $i$-линейных отображений; с каждой стороны последнего соотношения стоят $i+1$ пространств $V$. Теперь мы определим последовательно при любом $i>0$ пространства

$$
\begin{gathered}
\mathfrak{g}_{i}=\left\{X \in \operatorname{Hom}\left(\mathfrak{g}_{-1}, \mathfrak{g}_{i-1}\right) \mid X\left(v_{1}\right)\left(v_{2}, v_{3}, \ldots, v_{i+1}\right)=X\left(v_{2}\right)\left(v_{1}, v_{3}, \ldots, v_{i+1}\right),\right. \\
\left.v_{1}, \ldots, v_{i+1} \in \mathfrak{g}_{-1}\right\}
\end{gathered}
$$


Очевидно, что $\mathfrak{g}_{i} \subset S^{i+1}\left(\mathfrak{g}_{-1}^{*}\right) \otimes \mathfrak{g}_{-1}$, где $S^{i}$ - оператор $i$-й симметрической степени. Пусть $\mathfrak{g}_{0}$-модуль $\mathfrak{g}_{-1}$ точен, следовательно,

$$
\left(\mathfrak{g}_{-1}, \mathfrak{g}_{0}\right)_{*} \subset \mathfrak{v} \mathfrak{e c t}(n):=\mathfrak{d e r} \mathbb{C}\left[x_{1}, \ldots, x_{n}\right], \quad \text { где } \quad n=\operatorname{dim} \mathfrak{g}_{-1} .
$$

Легко проверить, что структура алгебры Ли на пространстве $\mathfrak{v e c t}(n)$ индуцирует структуру алгебры Ли на $\left(\mathfrak{g}_{-1}, \mathfrak{g}_{0}\right)_{*}$ (пространство $\left(\mathfrak{g}_{-1}, \mathfrak{g}_{0}\right)_{*}$ наделено структурой алгебры Ли, даже если $\mathfrak{g}_{-1}$ не является точным $\mathfrak{g}_{0}$-модулем, но в этом случае $\left(\mathfrak{g}_{-1}, \mathfrak{g}_{0}\right)$ нельзя вложить в $\mathfrak{v e c t}\left(\mathfrak{g}_{-1}^{*}\right)$, где $\mathfrak{v e c t}(V)$ - алгебра Ли полиномиальных векторных полей на линейном пространстве $V)$. Алгебра Ли $\left(\mathfrak{g}_{-1}, \mathfrak{g}_{0}\right)_{*}$ называется картановским продолжением пары $\left(\mathfrak{g}_{-1}, \mathfrak{g}_{0}\right)$. Картановское продолжение является алгеброй Ли симметрий $G$-структуры в пространстве $T_{m} M$.

Пусть $E^{i}$ - оператор $i$-й внешней степени, а $V^{*}$ - пространство, двойственное к $V$. Положим

$$
C_{\left(\mathfrak{g}_{-1}, \mathfrak{g}_{0}\right)_{*}}^{k, s}=\mathfrak{g}_{k-s} \otimes E^{s}\left(\mathfrak{g}_{-1}^{*}\right)
$$

Дифференциал $\partial_{s}: C_{\left(\mathfrak{g}_{-1}, \mathfrak{g}_{0}\right)_{*}}^{k, s} \longrightarrow C_{\left(\mathfrak{g}_{-1}, \mathfrak{g}_{0}\right)_{*}}^{k, s+1}$ задан формулой

$$
\left(\partial_{s} f\right)\left(v_{1}, \ldots, v_{s+1}\right)=\sum_{i}(-1)^{i}\left[f\left(v_{1}, \ldots, \hat{v}_{s+1-i}, \ldots, v_{s+1}\right), v_{s+1-i}\right]
$$

при любых $v_{1}, \ldots, v_{s+1} \in \mathfrak{g}_{-1}$, где, как обычно, букву с крышечкой следует проигнорировать. Как и задумано, $\partial_{s} \partial_{s+1}=0$, и гомологии этого бикомплекса называются когомологиями Спенсера пары $\left(\mathfrak{g}_{-1}, \mathfrak{g}_{0}\right)$ и обозначаются $H_{\left(\mathfrak{g}_{-1}, \mathfrak{g}_{0}\right)_{*}}^{k, s}$.

2.1. Предложение [34]. Структурные функиии $k$-го порядка $G$-структуръ составляют все препятствия $\kappa$ отождествлению $k$-й инфинитезимальной окрестности точки на многообразии с плоской $G$-структурой, с одной стороны, и k-ой инфинитезималъной окрестности данной точки $m$ данного многообразия $M$, c другой стороны. Для каждой точки $m$ эти структурные функиии линейно порождают пространство $H_{\left(\mathfrak{g}_{-1}, \mathfrak{g}_{0}\right)_{*}}^{k, 2}$. Эти препятствия определень, если все препятствия менвших порядков равны нулю.

2.2. Пример. Все структурные функции любой $G L(n)$-структуры тождественно обращаются в нуль. Действительно, поскольку $(V, \mathfrak{g l}(V))_{*} \simeq \operatorname{vect}(V)$, то $H^{2}\left(V ;(V, \mathfrak{g l}(V))_{*}\right) \simeq H^{2}(\mathfrak{g l}(V) ; V)=0$ по общей теореме о коиндуцированных модулях [53]. Это согласуется с тем, что по определению все $G L(n)$-структуры локально эквивалентны, в частности, локально плоски.

Ясно, что если $\left(\mathfrak{g}_{-1}, \mathfrak{g}_{0}\right)_{*}=\bigoplus_{i=-1}^{h} \mathfrak{g}_{i}$, то порядок структурной функции данной $G$-структуры может принимать значение от 1 до $h+2$ (или от 1 до $\infty$, если $h=\infty)$.

2.3. Пример. Пусть $\mathfrak{g}_{0}=\mathfrak{c o}(V):=\mathfrak{o}(V) \oplus \mathbb{C} z$ - алгебра Ли конформных преобразований $\mathfrak{g}_{-1}=V, \operatorname{dim} V=n$. Пусть $V=V_{1} \oplus V_{2}$ с базисом $\partial_{x}$ и $\partial_{y}$ при $n=2$ и 
пусть $\mathfrak{o}(V):=\mathbb{C}\left(x \partial_{x}-y \partial_{y}\right)$. Тогда (в дифференциальной геометрии это утверждение называется теоремой Луивилля [34])

$$
\begin{aligned}
(V, \mathfrak{c o}(V))_{*} & = \begin{cases}\mathfrak{v e c t}\left(V^{*}\right) & \text { для } n=1, \\
\mathfrak{v e c t}\left(V_{1}^{*}\right) \oplus \mathfrak{v e c t}\left(V_{2}^{*}\right) & \text { для } n=2, \\
V \oplus \mathfrak{c o}(V) \oplus V^{*} \simeq \mathfrak{o}(n+2) & \text { для } n>2 ;\end{cases} \\
(V, \mathfrak{o}(V))_{*} & = \begin{cases}V & \text { для } n=1, \\
V \oplus \mathfrak{o}(V) & \text { для } n \geqslant 2 .\end{cases}
\end{aligned}
$$

Отсюда мы сразу выводим теорему Леви-Чивита [54].

Теорема (Леви-Чивита). Значения тензора Римана на любом п-мерном римановом многообразии принадлежат пространству $H_{(V, \mathfrak{o}(V))_{*}}^{2,2}, a H_{(V, \mathfrak{o}(V))_{*}}^{1,2}=0$.

СлеДСТвиЕ. 1. В разложении метрики в ряд Тейлора в некоторой точке

$$
g(x)=\eta+s_{1} x+s_{2} x^{2}+s_{3} x^{3}+\cdots
$$

(здесь $\eta$ - каноническая форма; $x$ - вектор координат, $x^{2}$ - вектор пар координат и т.д.) член, содержащий $s_{1}$, может быть обращен в нуль заменой координат.

2. Поскольку нет структурных функиий порядка больше 2, все члены $s_{i}$ при $i \geqslant 2$ зависят лишь от тензора Римана.

Происхождение этого следствия непросто извлечь из стандартных учебников по дифференциальной геометрии, в то время как явный вид пространства $H^{2}\left(V,(V, \mathfrak{o}(V))_{*}\right)$ делает его очевидным.

2.4. Замечание (см. [32]). Пусть $H_{k}^{s}$ представляет собой компоненту степени $k$ в $H^{s}\left(\mathfrak{g}_{-1} ;\left(\mathfrak{g}_{-1}, \mathfrak{g}_{0}\right)_{*}\right)$ относительно $\mathbb{Z}$-градуировки, индуцированной $\mathbb{Z}$-градуировкой в алгебре Ли $\left(\mathfrak{g}_{-1}, \mathfrak{g}_{0}\right)_{*}$. Очевидно, что $H_{\left(\mathfrak{g}_{-1}, \mathfrak{g}_{0}\right)_{*}}^{k, s}=H_{k}^{s}$, так что

$$
\bigoplus_{k} H_{\left(\mathfrak{g}_{-1}, \mathfrak{g}_{0}\right)_{*}}^{k, s}=H^{s}\left(\mathfrak{g}_{-1} ;\left(\mathfrak{g}_{-1}, \mathfrak{g}_{0}\right)_{*}\right) .
$$

Это замечание сильно упрощает вычисления, если алгебра Ли $\left(\mathfrak{g}_{-1}, \mathfrak{g}_{0}\right)_{*}$ является простой и конечномерной: в этом случае мы можем применить теорему ББВ. В неголономном случае, который мы рассмотрим ниже, мы воспользуемся этим замечанием, чтобы дать компактное определение структурных функций. Мы можем в любой момент восстановить биградуировку, но работать с одной градуировкой значительно проще, и у нас меньше шансов ошибиться ${ }^{4)}$.

4) Ср. с проблемами, с которыми приходилось бороться в пионерских работах [13]-[15], где неголономные тензоры сначала вычислялись с помощью дифференциала, квадрат которого был отличен от нуля. Тензоры Вагнера не только не полностью описывают картину, но еще и выглядят ужасно [18], [31]. 


\section{3. СТРУКТУРНЫЕ ФУНКЦИИ НЕГОЛОНОМНЫХ СТРУКТУР}

Чтобы охватить структуры, подобные контактной структуре, мы должны немного обобщить понятие картановского продолжения: с касательным расслоением на любом неголономном многообразии естественным образом ассоциирован пучок градуированных нильпотентных алгебр Ли (ср. [55], [56]). Например, для любого нечетномерного многообразия с контактной структурой таким пучком является пучок гейзенберговых алгебр Ли.

3.1. Неголономные многообразия [3], [55]. Продолжения Танаки-Щепочкиной. Пусть $M^{n}-n$-мерное многообразие с неинтегрируемым распределением $\mathcal{D}$. Пусть

$$
\mathcal{D}=\mathcal{D}_{-1} \subset \mathcal{D}_{-2} \subset \mathcal{D}_{-3} \subset \cdots \subset \mathcal{D}_{-d}
$$

- последовательность строгих включений, где слой распределения $\mathcal{D}_{-i}$ в точке $x \in M$ есть

$$
\mathcal{D}_{-i+1}(x)+\left[\mathcal{D}_{-1}, \mathcal{D}_{-i+1}\right](x) .
$$

Здесь $\left[\mathcal{D}_{-1}, \mathcal{D}_{-i+1}\right]=\operatorname{Span}\left([X, Y] \mid X \in \Gamma\left(\mathcal{D}_{-1}\right), Y \in \Gamma\left(\mathcal{D}_{-i+1}\right)\right)$, а $d$ - наименьший номер такой, что

$$
\mathcal{D}_{-d}(x)+\left[\mathcal{D}_{-1}, \mathcal{D}_{-d}\right](x)=\mathcal{D}_{-d}(x)
$$

В случае $\mathcal{D}_{-d}=T M$ распределение называется вполне неголономным. Число $d=$ $d(M)$ называется степенъю неголономности. Многообразие $M$ с распределением $\mathcal{D}$ будем называть неголономным, если $d(M) \neq 1$. Положим

$$
n_{0}(x)=0 ; \quad n_{i}(x)=\operatorname{dim} \mathcal{D}_{-i}(x), \quad 1 \leqslant i<d ; \quad n_{d}(x)=n-n_{d-1} .
$$

Распределение $\mathcal{D}$ называется регулярным, если все размерности $n_{i}$ постоянны на $M$. Мы будем рассматривать лишь регулярные вполне неголономные распределения, удовлетворяющие, кроме того, некоторому условию транзитивности (6) (см. ниже).

Каждому касательному расслоению над неголономным многообразием $(M, \mathcal{D})$ мы сопоставим расслоение $\mathbb{Z}$-градуированных нильпотентных алгебр Ли следующим образом. Зафиксируем точку $\mathrm{pt} \in M$. Обычная фильтрация степенями максимального идеала $\mathfrak{m}:=\mathfrak{m}_{\mathrm{pt}}$, состоящего из функций, обращающихся в pt в нуль, должна быть модифицирована, потому что разные координаты могут иметь разные "степени". Распределение $\mathcal{D}$ индуцирует следующую фильтрацию на $\mathfrak{m}$ :

$$
\begin{aligned}
\mathfrak{m}_{k}=\{ & \left\{\in \mathfrak{m} \mid X_{1}^{a_{1}} \ldots X_{n}^{a_{n}}(f)=0 \quad \text { для всех } \quad X_{1}, \ldots, X_{n_{1}} \in \Gamma\left(\mathcal{D}_{-1}\right),\right. \\
& X_{n_{1}+1}, \ldots, X_{n_{2}} \in \Gamma\left(\mathcal{D}_{-2}\right), \ldots, X_{n_{d-1}+1}, \ldots, X_{n} \in \Gamma\left(\mathcal{D}_{-d}\right) \\
& \text { таких, что } \left.\sum_{1 \leqslant i \leqslant d} i\left(\sum_{n_{i-1}<j \leqslant n_{i}} a_{j}\right) \leqslant k\right\},
\end{aligned}
$$


где $\Gamma\left(\mathcal{D}_{-j}\right)-$ пространство ростков в точке pt сечений расслоения $\mathcal{D}_{-j}$. Теперь мы сопоставляем фильтрации

$$
\mathcal{D}=\mathcal{D}_{-1} \subset \mathcal{D}_{-2} \subset \mathcal{D}_{-3} \subset \cdots \subset \mathcal{D}_{-d}=T M
$$

ассоциированный градуированный пучок

$$
\operatorname{gr}(T M)=\oplus \operatorname{gr} \mathcal{D}_{-i}, \quad \text { где } \quad \operatorname{gr} \mathcal{D}_{-i}=\mathcal{D}_{-i} / \mathcal{D}_{-i+1},
$$

а скобка сечений из $\operatorname{gr}(T M)$ по определению индуцирована скобкой векторных полей (сечений расслоения $T M$ ). Мы требуем следующего условия транзитивности:

алгебры Ли $\left.\operatorname{gr}(T M)\right|_{\mathrm{pt}}$, индуцированные в каждой точке $\mathrm{pt} \in M$, изоморфны.

Градуировка координат определяет следующую нестандартную градуировку алгебры Ли $\mathfrak{v e c t}(n)$, см. (4):

$$
\begin{array}{r}
\operatorname{deg} x_{1}=\cdots=\operatorname{deg} x_{n_{1}}=1, \\
\operatorname{deg} x_{n_{1}+1}=\cdots=\operatorname{deg} x_{n_{2}}=2, \\
\ldots \ldots \cdots \cdots \cdots \cdot \ldots \cdot \ldots \cdot \operatorname{deg} x_{n}=d .
\end{array}
$$

Обозначим $\mathfrak{v}=\bigoplus_{i \geqslant-d} \mathfrak{v}_{i}$ алгебру Ли $\mathfrak{v e c t}(n)$ с градуировкой (7). Можно показать, что полное продолжение

$$
\left(\mathfrak{g}_{-}\right)_{*}:=\left(\mathfrak{g}_{-}, \tilde{\mathfrak{g}}_{0}\right)_{*} \subset \mathfrak{v}, \quad \text { где } \quad \tilde{\mathfrak{g}}_{0}:=\mathfrak{d} \mathfrak{e} \mathfrak{r}_{0} \mathfrak{g}_{-},
$$

алгебры Ли $\mathfrak{g}_{-}:=\bigoplus_{i<0} \mathfrak{g}_{i}$ сохраняет $\mathcal{D}$.

Для неголономных многообразий аналогом группы $G$ из понятия $G$-структуры, а точнее, аналогом ее алгебры Ли $\mathfrak{g}=\operatorname{Lie}(G)$, является пара $\left(\mathfrak{g}_{-}, \mathfrak{g}_{0}\right)$, где $\mathfrak{g}_{0}-$ подалгебра дифференцирований алгебры Ли $\mathfrak{g}_{-}$, сохраняющих ее $\mathbb{Z}$-градуировку, т.е. $\mathfrak{g}_{0} \subset \mathfrak{d e \mathfrak { r } _ { 0 }} \mathfrak{g}_{-}$. Если $\mathfrak{g}_{0}$ явно не указана, то мы предполагаем, что $\mathfrak{g}_{0}=\mathfrak{d e} \mathfrak{r}_{0} \mathfrak{g}_{-}$, т.е. $\mathfrak{g}_{0}$ - максимально возможная алгебра.

По данной паре $\left(\mathfrak{g}_{-}, \mathfrak{g}_{0}\right)$ мы строим ее продолжение Картана-Танаки-Щепочкиной (КТЩ-продолжение) как максимальную подалгебру $\left(\mathfrak{g}_{-}, \mathfrak{g}_{0}\right)_{*}=\bigoplus_{k \geqslant-d} \mathfrak{g}_{k}$ в $\mathfrak{v}$ с данной неположительной частью $\left(\mathfrak{g}_{-}, \mathfrak{g}_{0}\right)$. Явная конструкция алгебры $\left(\mathfrak{g}_{-}, \mathfrak{g}_{0}\right)_{*}$ дана в [16], [30], [41] и ниже.

3.2. Естественные базисы в $T_{m} M$ : векторы $D_{i}$ и $Q_{i}[30]$. Вершик и Гершкович показали в [55], что каждая неголономная структура $\mathcal{D}$ на $M$ определяет структуру $\mathbb{Z}$-градуированной нильпотентной алгебры Ли в $\operatorname{gr}(T M)$. Мы будем рассматривать лишь многообразия с транзитивным действием группы диффеоморфизмов многообразия $M$ на $T M$, т.е. многообразия, на которых выполняется условие (6).

Естественный базис в каждом касательном пространстве $T_{m} M$ к произвольному многообразию $M$ состоит из частных производных. Если многообразие $M$ снабжено неголономной структурой, то существует два типа естественных базисов в $\operatorname{gr}\left(T_{m} M\right)$. 
В физической литературе по суперсимметриям и супергравитации элементы этих двух базисов, которые порождают алгебру Ли $\operatorname{gr}\left(T_{m} M\right)$ и изоморфную ей алгебру, обозначаются символами $D_{i}$ и $Q_{i}$, соответственно.

Рассмотрим простейший пример. Пусть $\operatorname{dim} M=2 n+1$, и неголономная структура на $M$ задана контактной формой $\alpha=d t-\sum\left(p_{i} d q_{i}-q_{i} d p_{i}\right)$. Векторные поля, принадлежащие распределению $\mathcal{D},-$ это поля

$$
X=f \partial_{t}+\sum\left(g_{i} \partial_{q_{i}}+h_{i} \partial_{p_{i}}\right) \quad \text { такие, что } \quad \alpha(X)=f-\sum\left(p_{i} g_{i}-q_{i} h_{i}\right)=0 .
$$

$\mathrm{B}$ частности, ни $\partial_{q_{i}}$, ни $\partial_{p_{i}}$ не являются сечениями распределения $\mathcal{D}$ в отличие от

$$
D_{p_{i}}=\partial_{q_{i}}+p_{i} \partial_{t}, \quad D_{q_{i}}=\partial_{p_{i}}-q_{i} \partial_{t} .
$$

Такие $D_{p_{i}}$ и $D_{q_{i}}$ являются примерами базисных векторов $D$-типа. Они и их скобки порождают пространство сечений расслоения $\operatorname{gr}(T M)$ в каждой точке $m$. Допуская некоторую вольность речи, мы говорим, что $D$-векторы натягивают $T_{m} M$; то же относится к $Q$-векторам, определенным ниже.

Алгебра Ли, которая сохраняет $\mathcal{D}$, состоит из векторных полей $X$ таких, что

$$
L_{X}(\alpha) \equiv 0 \quad \bmod \alpha
$$

(здесь $L_{X}$ - это производная Ли вдоль $X$ ). Соответствующие векторные поля в нашем частном случае контактного распределения являются контактными векторными полями $K_{f}$ с производящей функцией $f \in \mathbb{C}[t, p, q]$ :

$$
K_{f}=(2-E)(f) \frac{\partial}{\partial t}-H_{f}+\frac{\partial f}{\partial t} E,
$$

где $E=\sum_{i} y_{i} \partial_{y_{i}}-$ onepamop Эйлера (здесь $y_{i}$ - все координаты, кроме $t$ ), а $H_{f}-$ гамильтоново поле с гамильтонианом $f$, сохраняющее $d \alpha$ :

$$
H_{f}=\sum_{i \leqslant n}\left(\frac{\partial f}{\partial p_{i}} \frac{\partial}{\partial q_{i}}-\frac{\partial f}{\partial q_{i}} \frac{\partial}{\partial p_{i}}\right) .
$$

Легко проверить, что

$$
L_{K_{f}}(\alpha)=2 \frac{\partial f}{\partial t} \alpha
$$

Базис в касательном пространстве состоит из элементов $K_{p_{i}}=\partial_{q_{i}}-p_{i} \partial_{t}, K_{q_{i}}=$ $\partial_{p_{i}}+q_{i} \partial_{t}$ и их скобки. Эти поля $K_{p_{i}}$ и $K_{q_{i}}$ являются полями базисных векторов $Q$-типа.

Как интерпретировать векторы $D$-типа и $Q$-типа? Пусть $\mathfrak{n}=\bigoplus_{-d \leqslant i \leqslant-1} \mathfrak{n}_{i}-$ нильпотентная алгебра Ли, порожденная пространством $\mathfrak{n}_{-1}$. Пусть $B=\left\{b_{1}, \ldots, b_{n}\right\}-$ градуированныц базис в $\mathfrak{n}$, другими словами, первые $n_{1}:=\operatorname{dim} \mathfrak{n}_{-1}$ элементов порождают $\mathfrak{n}_{-1}$, следующие $n_{2}-n_{1}:=\operatorname{dim} \mathfrak{n}_{-2}$ элементов порождают $\mathfrak{n}_{-2}$ и т.д. Пусть $N$ - связная односвязная группа Ли с алгеброй Ли $\mathfrak{n}$. Рассмотрим две системы векторных полей на $N$ : левоинвариантные поля $D_{i}$ и правоинвариантные поля $Q_{i}$ такие, что

$$
D_{i}(e)=Q_{i}(e)=b_{i} \quad \text { при всех } i=1, \ldots, n
$$


$(e-$ единица группы $N)$.

Отметим, что мы отходим от соглашений, принятых в физических работах, где символы $D_{i}$ и $Q_{i}$ относятся лишь к первым $n_{1}$ элементам, порождающим алгебру Ли $\mathfrak{n}$.

Пусть $\mathfrak{g}_{-}$- такая реализация алгебры Ли $\mathfrak{n}$ левоинвариантными векторными полями, что векторные поля $D_{i}$ порождают $\mathfrak{g}_{-}$(или, что то же самое, алгебру $\mathfrak{n}$ ). Пусть $\theta^{i}$ - правоинвариантные 1 -формы на $N$ такие, что $\theta^{i}\left(Q_{j}\right)=\delta_{j}^{i}$. Теперь любое векторное поле $X$ на $N$ имеет вид

$$
X=\sum_{i=1}^{n} \theta^{i}(X) Q_{i} .
$$

Поскольку каждое поле $D_{i}$ коммутирует с каждым полем $Q_{j}($ если $\mathfrak{n}-$ супералгебра Ли, они суперкоммутируют [30]), то

$$
\theta^{i}\left(\left[D_{j}, X\right]\right)=D_{j}\left(\theta^{i}(X)\right) .
$$

Теперь определим правоинвариантное распределение $\mathcal{D}$ на $N$ такое, что $\left.\mathcal{D}\right|_{e}=$ $\mathfrak{n}_{-1}$. Ясно, что распределение $\mathcal{D}$ выделено в $T N$ следующей системой уравнений на поле $X \in \mathfrak{v e c t}(n)$ :

$$
\theta^{n_{1}+1}(X)=0, \quad \ldots, \quad \theta^{n}(X)=0 .
$$

Поскольку каждое поле $D_{i}$ коммутирует с каждым полем $Q_{j}$, алгебра Ли $\mathfrak{g}_{-}$ сохраняет $\mathcal{D}$. Координаты на $N$, градуировка которых описана в $(7)$, задают два вложения алгебры Ли $\mathfrak{n}$ в $\mathfrak{v e c t}(n)$ : одно натянуто на поля $D_{i}$, а другое - на поля $Q_{i}$.

Обозначим $\mathfrak{g}=\bigoplus_{i \geqslant-d} \mathfrak{g}_{i}$ алгебру Ли $\mathfrak{v e c t}(n)$ с градуировкой (7). Тогда алгебра Ли $\mathfrak{g}_{-}=\bigoplus_{i<0} \mathfrak{g}_{i}$ сохраняет распределение $\mathcal{D}$. Позже мы покажем, что полное продолжение алгебры Ли $\mathfrak{g}_{-}$, т.е. $\left(\mathfrak{g}_{-}\right)_{*}:=\left(\mathfrak{g}_{-}, \tilde{\mathfrak{g}}_{0}\right)_{*}$, где $\tilde{\mathfrak{g}}_{0}:=\mathfrak{d} \mathfrak{r}_{0} \mathfrak{g}_{-}$, тоже сохраняет распределение $\mathcal{D}$ и по построению является максимальной алгеброй Ли, сохраняющей $\mathcal{D}$.

Итак, мы видим, что с каждым неголономным многообразием $(M, \mathcal{D})$ ассоциирована некоторая $G$-структура, причем алгебра Ли группы $G$ есть $\operatorname{Lie}(G)=\mathfrak{d e r}_{0}\left(\mathfrak{g}_{-}\right)$. Но структурные функции этой $G$-структуры совершенно не отражают неголономный характер многообразия $M$.

Напомним в этой связи пример из [34]. Пусть $W_{1} \subset W$ - подпространство размерности $k$, а $G \subset G L(W)$ - параболическая подгруппа, сохраняющая это подпространство. Тогда задать $G$-структуру на многообразии $M$, где $\operatorname{dim} M=\operatorname{dim} W$, означает задать дифференциальную $k$-систему или $k$-мерное распределение. Фиксированный репер $f$ в $T_{m} M$ задает изоморфизм $f: W \rightarrow T_{m} M$. Имея $G$-структуру на $M$, мы полагаем $\mathcal{D}(m)=f\left(W_{1}\right)$. Поскольку группа $G$ сохраняет $W_{1}$, то подпространства $\mathcal{D}(m)$ действительно зависят лишь от точки $m$, а не от $f$.

И наоборот, имея распределение $\mathcal{D}$, рассмотрим реперы $f$ такие, что $f^{-1}(\mathcal{D}(m))=$ $W_{1}$. Они образуют $G$-структуру. Плоская $G$-структура соответствует интегрируемым распределениям. 
Чтобы учесть неголономную природу многообразия $M$, нам дополнительно потребуется аналог предложения 2.1 в случае, когда естественный базис в касательном пространстве состоит не из частных производных, а из ковариантных производных, отвечающих связности, заданной теми же уравнениями Пфаффа, которые определяют наше неголономное распределение. Поэтому вместо $T_{m} M=\mathfrak{g}_{-1}$ мы должны взять $(\operatorname{gr}(T M))_{m}=\mathfrak{g}_{-}$. Чтобы сформулировать такое предложение, нам нужно

1) определить простейшую неголономную структуру - плоскую неголономную структуру;

2) определить аналог алгебры Ли $\mathfrak{g}_{0}$ в том случае, когда пространство $\mathfrak{g}_{-1}$ заменено на алгебру $\mathfrak{g}_{-}$и задано лишь распределение;

3) определить аналог алгебры Ли $\left(\mathfrak{g}_{-1}, \mathfrak{g}_{0}\right)_{*}$;

4) определить аналог инвариантов $H_{\left(\mathfrak{g}_{-1}, \mathfrak{g}_{0}\right)_{*}}^{k, 2}$.

Мы решаем поставленные проблемы следующим образом.

1. Пусть $\mathfrak{n}=\bigoplus_{-d \leqslant i \leqslant-1} \mathfrak{n}_{i}-$ нильпотентная $\mathbb{Z}$-градуированная алгебра Ли, а $N$ - связная односвязная группа Ли с алгеброй Ли $\mathfrak{n}$ (как гладкое многообразие эта группа диффеоморфна пространству $\mathbb{C}^{r}$, где $\left.r=\operatorname{dim} \mathfrak{n}\right)$. Выберем в пространстве $\mathfrak{n}$ репер $f$, согласованный с $\mathbb{Z}$-градуировкой. Отождествим касательное пространство $T_{n} N$ в каждой точке $n$ с алгеброй Ли $\mathfrak{n}$ с помощью левого сдвига на элемент $n$ и рассмотрим в каждой точке $n$ множество всех реперов, получающихся из $f$ действием группы $G_{0}=\mathrm{Aut}_{0} \mathfrak{n}$ автоморфизмов алгебры Ли $\mathfrak{n}$, сохраняющих градуировку. В результате мы получим $G_{0}$-структуру на $N$. Эту структуру мы и будем называть простейшей неголономной структурой (плоской неголономной структрой), связанной с алгеброй Ли $\mathfrak{n}$.

Иначе говоря, пусть $\mathcal{D}$ - неголономное транзитивное (т.е. удовлетворяющее условию (6)) распределение на $M$, и пусть $\left.\operatorname{gr}(T M)\right|_{\mathrm{pt}} \cong \mathfrak{n}$. Пусть $F$ - флаг, который определяет распределение $\mathcal{D}$ в каждой точке $m \in M$. Пусть $N:=\mathbb{C}^{r}$ с фиксированным флагом $F$ и фиксированным репером $f$. Отождествив $T_{n} N$ с $N$ с помощью сдвигов на элементы $n$ нильпотентной группы Ли $N$, алгебра Ли которой есть $\mathfrak{g}_{-}$(поскольку группа $N$ некоммутативна, то мы выберем, скажем, левые сдвиги), мы зафиксируем репер и флаг - образы $f$ и $F$, соответственно, в каждом пространстве $T_{n} N$. Плоской неголономной структурой на $N$ назовем пару расслоений (расслоение реперов, распределение $\mathcal{D})$; слои первого и второго расслоений над точкой $n$ получаются соответственно из фиксированного репера и флага с помощью $G_{0}$-действия, где $G_{0}$ - это (связная и односвязная) группа Ли с алгеброй Ли $\mathfrak{g}_{0}$, определенной на следующем шаге.

2. Если задано только распределение $\mathcal{D}$, то положим $\mathfrak{g}_{0}:=\mathfrak{d e r} \mathfrak{r}_{0} \mathfrak{g}_{-}$. Часто интересно рассмотреть дополнительные структуры на распределении (скажем, риманову, см. [3], как в случае с метрикой Карно-Каратеодори), тогда $\mathfrak{g}_{0}$ есть подалгебра в $\mathfrak{d e r} \mathfrak{r}_{0} \mathfrak{g}_{-}$, в нашем примере $\mathfrak{d e r} \mathfrak{r}_{0} \mathfrak{g}_{-} \cap \mathfrak{o}\left(\mathfrak{g}_{-1}\right)$.

3. По определенной выше паре $\left(\mathfrak{g}_{-}, \mathfrak{g}_{0}\right)$ построим ее $k$-е КТЩ-продолжение при $k>0$, положив

$$
\mathfrak{g}_{k}=\left(i\left(S^{\bullet}\left(\mathfrak{g}_{-}^{*}\right) \otimes \mathfrak{g}_{-}\right) \cap j\left(S^{\bullet}\left(\mathfrak{g}_{-}^{*}\right) \otimes \mathfrak{g}_{0}\right)\right)_{k},
$$


где индекс $k$ выделяет компоненту степени $k, S^{\bullet}=\bigoplus S^{i}$ и

$$
\begin{aligned}
& i: S^{k+1}\left(\mathfrak{g}_{-}^{*}\right) \otimes \mathfrak{g}_{-} \longrightarrow S^{k}\left(\mathfrak{g}_{-}^{*}\right) \otimes \mathfrak{g}_{-}^{*} \otimes \mathfrak{g}_{-}, \\
& j: S^{k}\left(\mathfrak{g}_{-}^{*}\right) \otimes \mathfrak{g}_{0} \longrightarrow S^{k}\left(\mathfrak{g}_{-}^{*}\right) \otimes \mathfrak{g}_{-}^{*} \otimes \mathfrak{g}_{-}
\end{aligned}
$$

суть естественные вложения.

Аналогично случаю, когда $\mathfrak{g}_{-}-$коммутативная алгебра Ли, определим $\left(\mathfrak{g}_{-}, \mathfrak{g}_{0}\right)_{*}$ как $\bigoplus_{-d \leqslant k \leqslant h} \mathfrak{g}_{k}$, где пространство $\mathfrak{g}_{k}$ при $k>0$ задано формулой (13); тогда, как легко проверить, $\left(\mathfrak{g}_{-}, \mathfrak{g}_{0}\right)_{*}$ - алгебра Ли.

4. Рассуждения, аналогичные тем, что приведены в книге [34], показывают, что $H^{2}\left(\mathfrak{g}_{-} ;\left(\mathfrak{g}_{-}, \mathfrak{g}_{0}\right)_{*}\right)$ есть пространство значений неголономных структурных функций. Эти структурные функции составляют все препятствия к отождествлению инфинитезимальной окрестности точки $m$ на многообразии $M$ с неголономной структурой (заданной парой $\mathfrak{g}_{-}$и $\mathfrak{g}_{0}$ ) с инфинитезимальной окрестностью данной точки на плоском неголономном многообразии с теми же $\mathfrak{g}_{-}$и $\mathfrak{g}_{0}$.

Пространство $H^{2}\left(\mathfrak{g}_{-} ;\left(\mathfrak{g}_{-}, \mathfrak{g}_{0}\right)_{*}\right)$ естественно распадается на однородные компоненты, степени которых будут называться порядками структурных функиий. Степени пробегают значения от $2-d$ до $h+2 d$ или до $\infty$, если $h=\infty$. Как и в случае коммутативной алгебры Ли $\mathfrak{g}_{-}=\mathfrak{g}_{-1}$, структурные функции порядка $k$ можно интерпретировать как препятствия к плоскостности неголономного многообразия с $\left(\mathfrak{g}_{-}, \mathfrak{g}_{0}\right)$-структурой при условии, что препятствия меньших порядков обращаются в нуль. Заметим, что для неголономных многообразий порядок структурной функции не имеет больше прямой связи с порядком инфинитезимальной окрестности точек, которые мы хотим отождествить, поскольку разные частные производные имеют разные "степени".

Разные фильтрованные алгебры Ли $L$ с одной и той же ассоциированной градуированной алгеброй $\mathfrak{g}_{-}:=\operatorname{gr} L$ описываются в точности теми кограницами, которые ответственны за фильтрованные деформации алгебры $\mathfrak{g}_{-}$. При переходе к когомологиям все они обращаются в нуль, поэтому приведенные выше структурные функции корректно определены.

\section{4. ТЕНЗОРЫ РИМАНА И ВЕЙЛЯ. ПРОЕКТИВНЫЕ СТРУКТУРЫ}

4.1. Конформный случай. Для классических областей $X=S / P$, которые рассмотрел Гончаров, структурные функции являются обобщениями тензора Вейля - конформно-инвариантной части тензора Римана для $S=O(n+2)$ и $G=C O(n)$. В большинстве случаев, рассмотренных Гончаровым, имеет место равенство

$$
\left(\mathfrak{g}_{-1}, \mathfrak{g}_{0}\right)_{*}=\mathfrak{s}:=\operatorname{Lie}(S),
$$

и описание структурных функций сводится к частному случаю теоремы ББВ. В частности, если выполнено равенство (14), то пространство $H^{2}\left(\mathfrak{g}_{-1} ;\left(\mathfrak{g}_{-1}, \mathfrak{g}_{0}\right)_{*}\right)$, рассматриваемое как $\mathfrak{g}_{0}$-модуль, имеет столько же неприводимых компонент и ту же размерность, что и пространство $E^{2}\left(\mathfrak{g}_{-1}\right)$, а различаются эти пространства лишь весами. 
4.2. Обобщенный риманов случай. После того как мы редуцировали алгебру Ли $\mathfrak{g}_{0}$, сохранив лишь ее полупростую часть $\widehat{\mathfrak{g}_{0}}$ и профакторизовав по центру, мы не можем непосредственно применить теорему ББВ, поскольку алгебра $\left(\mathfrak{g}_{-1}, \widehat{\mathfrak{g}_{0}}\right)_{*}=\mathfrak{g}_{-1} \oplus \widehat{\mathfrak{g}_{0}}$ не является простой. Но мы можем все-таки свести задачу к конформному случаю, поскольку, как известно,

$$
H^{2}\left(\mathfrak{g}_{-1} ;\left(\mathfrak{g}_{-1}, \widehat{\mathfrak{g}_{0}}\right)_{*}\right)=H^{2}\left(\mathfrak{g}_{-1} ; \mathfrak{s}\right) \oplus S^{2}\left(\mathfrak{g}_{-1}^{*}\right) .
$$

В неголономном случае сходная редукция достигается применением теоремы Премета, сформулированной ниже. Общий случай этой теоремы достаточно явно формулируется, но не так прост, как (15). Однако, хотя соответствующий аналог равенства (15), а именно

$$
H^{2}\left(\mathfrak{g}_{-} ;\left(\mathfrak{g}_{-}, \widehat{\mathfrak{g}_{0}}\right)_{*}\right)=H^{2}\left(\mathfrak{g}_{-} ; \mathfrak{s}\right) \oplus S^{2}\left(\mathfrak{g}_{-1}^{*}\right)
$$

не всегда имеет место, он верен во многих случаях, представляющих интерес (при "контактной” градуировке).

4.3. Проективный случай. Теоремы Ямагучи и Серра. Если соотношение (14) не выполняется, алгебра Ли $\mathfrak{s}$ является собственной подалгеброй в $\left(\mathfrak{g}_{-1}, \mathfrak{g}_{0}\right)_{*}$. Интересно поэтому, во-первых, перечислить случаи, когда, начав с простой супералгебры Ли $\mathfrak{s}=\bigoplus_{i \geqslant-d} \mathfrak{s}_{i}$, мы получаем прямой аналог равенства (14)

$$
\left(\mathfrak{s}_{-}, \mathfrak{s}_{0}\right)_{*}=\mathfrak{s}
$$

во-вторых, найти, что́ является "полным продолжением" алгебры Ли $\mathfrak{s}_{-}$, т.е. вычислить $\left(\mathfrak{s}_{-}\right)_{*}:=\left(\mathfrak{s}_{-}, \tilde{\mathfrak{s}}_{0}\right)_{*}$, где $\tilde{\mathfrak{s}}_{0}:=\mathfrak{d e r} \mathfrak{r}_{0} \mathfrak{s}_{-}$.

Для простых конечномерных алгебр Ли $\mathfrak{s}$ Ямагучи приводит ответ [16], представляющий интерес для нас, и мы его воспроизведем. Ответ для простых супералгебр Ли получен Щепочкиной (не опубликовано).

КоммеНТАРИй. Естественно ожидать, что алгебра $\tilde{\mathfrak{s}}_{0}$ строго содержит алгебру $\mathfrak{s}_{0}$, и, следовательно, $\left(\mathfrak{s}_{-}\right)_{*}$ должна строго содержать $\mathfrak{s} ;$ однако на самом деле они совпадают, в частности, $\tilde{\mathfrak{s}}_{0}=\mathfrak{s}_{0}$.

4.3.1. Теорема (Ямагучи, [16]). Равенство $\left(\mathfrak{s}_{-}\right)_{*}=\mathfrak{s}$ верно всегда за исключением следующих случаев:

1) если $\mathfrak{s}$ - алгебра с градуировкой глубины $d=1\left(\right.$ в этом случае $\left.\left(\mathfrak{s}_{-}\right)_{*}=\mathfrak{v e c t}\left(\mathfrak{s}_{-}^{*}\right)\right)$;

2) если $\mathfrak{s}$ - алгебра с градуировкой глубины $d=2$, причем $\operatorname{dim} \mathfrak{s}_{-2}=1$, m.е. с "контактной" градуировкой; в этом случае $\left(\mathfrak{s}_{-}\right)_{*}=\mathfrak{k}\left(\mathfrak{s}_{-}^{*}\right)$; это отвечает выбору тех вершин диаграмм Дынкина, которые соединень с верииной, отвечающей максимальному корню на расширенной диаграмме;

3) если $\mathfrak{s}=\mathfrak{s l}(n+1)$ или $\mathfrak{s}=\mathfrak{s p}(2 n)$ с градуировкой, отвечающей выбору первого $u$-го из простых кокорней, где $1<i<n$ для $\mathfrak{s l}(n+1)$ u $i=n$ для $\mathfrak{s p}(2 n)$; в этом случае $d=2$, причем $\operatorname{dim} \mathfrak{s}_{-2}>1 \partial л я \mathfrak{s l}(n+1), u d=3 \partial л я \mathfrak{s p}(2 n)$.

Более того, равенство $\left(\mathfrak{s}_{-}, \mathfrak{s}_{0}\right)_{*}=\mathfrak{s}$ тоже верно почти всегда. Случаи, когда равенство нарушается (и возможно проективное действие) - это случаи алгебр 
$\mathfrak{s l}(n+1)$ и $\mathfrak{s p}(2 n)$ с градуировкой, заданной выбором лишь одного (первого) простого кокорня.

Случай 1 теоремы Ямагучи: конформный (вейлев) случай разобран в [32], риманов случай - в [43].

Для классических областей $X=S / P$ равенство (14) неверно лишь для групп $S=S L(n+1)$ и $X=\mathbb{C} P^{n} ;$ при этом $\mathfrak{g}_{0}=\mathfrak{g l}(n)$ и $\left(\mathfrak{g}_{-1}, \mathfrak{g}_{0}\right)_{*}=\mathfrak{v e c t}(n)$. Пространство “тотальных" структурных функций $H^{2}\left(\mathfrak{g}_{-1} ; \mathfrak{v e c t}(n)\right)$ отличается от $H^{2}\left(\mathfrak{g}_{-1} ; \mathfrak{s}\right)$, структурные функции в последнем случае отвечают препятствиям к проективной структуре, многочисленные аспекты которой описаны в [57], [58].

Риманова версия этого проективного случая соответствует алгебрам $\widehat{\mathfrak{g}_{0}}=\mathfrak{s l}(n)$ и $\left(\mathfrak{g}_{-1}, \widehat{\mathfrak{g}_{0}}\right)_{*}=\mathfrak{s v e c t}(n)$, т.е. алгебре Ли бездивергентных векторных полей.

Случаи полного продолжения $\left(\mathfrak{s}_{-1}\right)_{*}=\mathfrak{v e c t}\left(\mathfrak{s}_{-1}^{*}\right)$ и их римановы версии $\left(\mathfrak{s}_{-1}\right)_{*}=$ $\mathfrak{s v e c t}\left(\mathfrak{s}_{-1}^{*}\right)$, также как и $\left(\mathfrak{s}_{-1}\right)_{*}=\mathfrak{h}\left(\mathfrak{s}_{-1}^{*}\right)$, рассмотрел давным-давно Серр [34] и доказал следующую теорему.

4.3.2. Теорема (Серр, [34]; суперверсии теоремы см. в [43], [59]). Справедливъь следующие утверждения:

$$
\begin{aligned}
& H^{2}\left(\mathfrak{s}_{-1} ; \mathfrak{v e c t}(n)\right)=0, \quad H^{2}\left(\mathfrak{s}_{-1} ; \mathfrak{s v e c t}(n)\right)=0 ; \\
& H^{2}\left(\mathfrak{s}_{-1} ; \mathfrak{h}(2 n)\right)=E^{3}\left(\mathfrak{s}_{-1}^{*}\right) .
\end{aligned}
$$

Случай 2 теоремы Ямагучи охвачен теоремой Премета и формулой (18) (обе приведены ниже).

Случай 3 теоремы Ямагучи рассмотрен в разделе 7 данной работы.

Всюду ниже для многообразий $X=S / P$ с неголономными структурами мы называем тензоры неголономными вейлевыми или неголономными конформными, если они отвечают когомологиям алгебр Ли $\mathfrak{g}_{-}$с коэффициентами в $\left(\mathfrak{g}_{-}, \mathfrak{g}_{0}\right)_{*}$, и говорим о неголономном римановом тензоре для неголономных структурных функций алгебры Ли $\left(\mathfrak{g}_{-}, \widehat{\mathfrak{g}_{0}}\right)_{*}$, где $\widehat{\mathfrak{g}_{0}}-$ полупростая часть алгебры Ли $\mathfrak{g}_{0}$, а также о неголономном проективном тензоре для когомологий с коэффициентами в $\mathfrak{s}=$ $\operatorname{Lie}(S)$ во всех случаях, когда $\mathfrak{s}-$ подалгебра в $\left(\mathfrak{g}_{-}, \mathfrak{g}_{0}\right)_{*}$ (например, для частичных КТЩ-продолжений, см. [38]).

4.4. Простейшие примеры (выбор первого простого кокорня в $\mathfrak{s p}(2 n+2)$ ). Пусть $\mathfrak{g}_{-}=\mathfrak{h} \mathfrak{e}(2 n)$ - гейзенбергова алгебра Ли. Тогда $\mathfrak{g}_{0}=\mathfrak{c s p}(2 n)($ т.е. $\mathfrak{s p}(2 n) \oplus \mathbb{C} z)$, a $\left(\mathfrak{g}_{-}, \mathfrak{g}_{0}\right)_{*}$ - алгебра Ли $\mathfrak{k}(2 n+1)$ контактных векторных полей.

До сих пор никто не предложил аналог теоремы Серра для инволютивности простых $\mathbb{Z}$-градуированных супералгебр Ли глубины больше 1 (см. [43]). Примеры из [59] показывают, что если такой аналог и существует, то теорема будет гораздо более замысловатой.

Поскольку

$$
H^{2}(\mathfrak{h} \mathfrak{e}(2 n) ; \mathfrak{k}(2 n+1))=0,
$$

то понятно, почему Пфаффово уравнение $\alpha(X)=0$ на поле $X \in \mathfrak{v e c t}(2 n+1)$ можно привести к каноническому виду (см. [60]). Равенство (18) есть несложное следствие утверждения о когомологиях коиндуцированных модулей [53]. Для неголономного 
риманова тензора в этом случае мы видим, что $\widehat{\mathfrak{g}_{0}}=\mathfrak{s p}(2 n)$, а $\left(\mathfrak{g}_{-}, \widehat{\mathfrak{g}_{0}}\right)_{*}-$ пуассонова алгебра Ли $\mathfrak{p o}(2 n)$, натянутая на поля $K_{f}$, где $\partial f / \partial t=0$.

Более того, из равенства (18) и короткой точной последовательности

$$
0 \longrightarrow \mathfrak{p o}(2 n) \longrightarrow \mathfrak{k}(2 n+1) \stackrel{\frac{\partial}{\partial t}: K_{f} \mapsto \frac{\partial f}{\partial t}}{\longrightarrow} \mathbb{C}[t, p, q] \longrightarrow 0
$$

мы легко выводим с помощью соответствующей длиной точной последовательности, что $H^{2}(\mathfrak{h e i}(2 n) ; \mathfrak{p o}(2 n))=0$. В наших терминах этот факт (обычно называемый теоремой Дарбу и доказываемый аналитическими средствами [60]) является объяснением того, почему контактную форму $\alpha$ можно привести к каноническому виду не только в каждой точке, но и локально.

4.5. Другие примеры. Многочисленные примеры неголономных проективных структур в различных ситуациях приведены в [21]-[25], [17] и (в суперокружении) в книге [37]. Вооружившись пакетом SuperLie, мы можем теперь легко сосчитать соответствующие когомологии алгебр Ли. Теоремы Премета говорят, что́ же именно нужно сосчитать в неголономном римановом случае, и снова для SuperLie это не составит труда: мы приведем лишь несколько примеров (один избранный кокорень для каждой простой алгебры $\mathfrak{s}$ и два избранных кокорня для двух серий одного из случаев Ямагучи).

\section{5. ТЕОРЕМЫ ПРЕМЕТА}

В 1990 г. Лейтес спросил Александра Премета, как свести вычисления пространства значений неголономного тензора Римана к неголономному тензору Вейля аналогично формуле (15), а именно верно ли, что формула (16) всегда справедлива?

Премет написал два письма с общим ответом. Одно письмо воспроизведено практически без изменений ниже (Д.Л. отвечает, конечно же, за все ошибки, которые остались или были добавлены). В этом письме показано, как свести проблему вычисления первых когомологий алгебры Ли $\mathfrak{g}_{-}$с коэффициентами в некоторых $\mathfrak{g}_{-}$-модулях, не являющихся $\mathfrak{g}$-модулями, к теореме ББВ, что в некоторых случаях удается сделать. О таких когомологиях было известно крайне мало, исключение составляют теоремы Костанта (для $H^{1}$ ) и Лежера-Люкса (для $H^{2}$ ), причем в обеих теоремах рассматривается случай, когда $\mathfrak{g}_{-}$- максимальная нильпотентная подалгебра. В другом письме Премета (воспроизведенном в [61]) содержится мощное обобщение этих теорем на $H^{i}$ при любых $i$ и любых $\mathfrak{g}_{-}$.

Однако в неголономном случае вывести явный ответ из теоремы ББВ "руками" трудно, а дополнительные члены в римановом случае (см. пункт 5.4 ниже) требуют дополнительной работы. Поэтому теоремы Премета были отложены в сторону на 13 лет. Теперь, когда пакет программ SuperLie [19], первоначально предназначенный для супергравитационных целей, достаточно развит, мы в состоянии дать явный ответ (см. следующий раздел). В случаях, которые мы здесь рассматриваем (для максимальных параболических подалгебр), вычисления занимают несколько минут. Однако требуется гораздо больше времени, чтобы задокументировать результаты, 
да еще немало времени уходит на то, чтобы их интерпретировать, скажем, как в [43]. $\mathrm{K}$ нашему сожалению, Премет рассматривает свои теоремы как чисто техническое упражнение ("простая работа для Костанта"), недостаточно интересное для того, чтобы принять участие в этой статье в качестве соавтора.

5.1. Терминологические соглашения. Пусть $\mathfrak{g}-$ простая (конечномерная) алгебра Ли. Пусть $L^{\lambda}$ - неприводимый (конечномерный) g-модуль со старшим весом $\lambda$. Для произвольного $\mathfrak{g}$-модуля $E$ через $E_{\mu}$ обозначено его весовое подпространство веса $\mu$.

Пусть $R$ - корневая система алгебры Ли $\mathfrak{g}$, а $B$ - система простых корней в $R$. Пусть $W=W(R)$ - группа Вейля алгебры Ли $\mathfrak{g}$, a $l(w)$ - длина элемента $w \in W$; пусть $W_{i}$ - подмножество элементов длины $i$. Пусть $R_{I} \subset R$ и $B_{I}$ - система простых корней в $R_{I}$. Положим (отсюда также следует определение числа $k(i)$ )

$$
W(I)_{i}=\left\{w_{i, 1}, \ldots, w_{i, k(i)} \in W_{i} \mid w_{i, j}^{-1}\left(B \backslash B_{I}\right)>0 \quad \text { для всех } \quad 1 \leqslant j \leqslant k(i)\right\} .
$$

В качестве примера диаграммы Дынкина, отвечающей $B$, возьмем

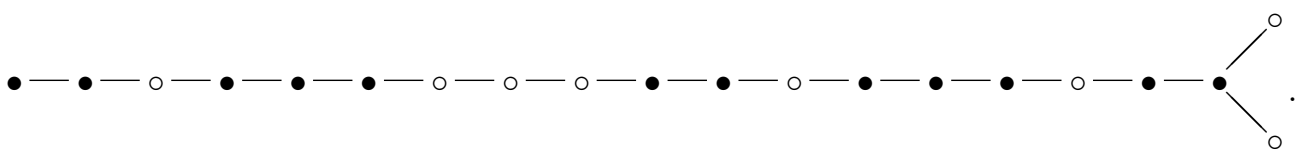

Пусть $B_{I}$ состоит из корней, отвечающих черным кружочкам. Представим $B_{I}$ как объединение связанных подграфов:

$$
B_{I}=B_{I}^{(1)} \coprod \cdots \coprod B_{I}^{(s)}
$$

где $s$ (в нашем примере $s=5$ ) - число связных компонент графа Дынкина $D_{I}$ системы $B_{I}$, а $B_{I}^{(i)}$ отвечает $i$-й связной компоненте графа $D_{I}$ (компоненты считаются слева направо). Положим

$$
c=\operatorname{card} B, \quad c_{i}=\operatorname{card}\left\{\alpha \in B \backslash B_{I} \mid\left(\alpha, B_{I}^{(i)}\right) \neq 0\right\}-1 .
$$

Ясно, что если $B_{I} \neq B$, то $c_{i} \in\{0,1,2\}$. Например, для графа алгебры Ли $\mathfrak{o}(40)$, изображенного выше, имеем

$$
c=20, \quad c_{1}=0, \quad c_{2}=1, \quad c_{3}=1, \quad c_{4}=1, \quad c_{5}=2 .
$$

Следующее утверждение очевидно.

УтвеРЖДЕНИЕ. 1) Равенство $c_{i}=2$ верно тогда и толъко тогда, когда $R$ имеет один из типов $D_{n}, E_{6}, E_{7}$ или $E_{8}$; одна из концевых вершин графа $D_{I}^{(i)}$ является точкой ветвления графа $D$, а оставшиеся концевые вершины графа $D_{I}^{(i)}$ не являются концевыми вериинами графа $D$;

2) равенство $c_{i}=0$ верно тогда и только тогда, когда все вериины графа системъ $B_{I}^{(i)}$, кроме одной концевой, являются концевыми вериинами графа системы $R$. 
5.2. Теорема Ботта-Бореля-Вейля. Пусть $\mathrm{rkg}=r>1, I \subset\{1, \ldots, r\} ; \mathfrak{p}=$ $\mathfrak{p}_{I}$ - параболическая подалгебра, порожденная образующими Шевалле $X_{i}^{ \pm}$алгебры Ли $\mathfrak{g}$ за исключением образующих $X_{k}^{+}$, где $k \in I$. Как известно, $\mathfrak{p}=\mathfrak{g}_{-} \ni \mathfrak{l}$, где $\mathfrak{l}$ - полупростая подалгебра Леви, порожденная всеми $X_{i}^{ \pm}$, кроме тех, для которых $i \notin I$. Ясно, что $\mathfrak{l}=\mathfrak{l}^{(1)} \oplus \mathfrak{z}$, где $\mathfrak{l}^{(1)}-$ производная алгебра алгебры Ли $\mathfrak{l}$, а $\mathfrak{z}=\mathfrak{z}(\mathfrak{l})-$ центр алгебры Ли l. Поэтому (в терминах раздела 4) $\mathfrak{g}_{0}=\mathfrak{l}$, а $\widehat{\mathfrak{g}_{0}}=\mathfrak{l}^{(1)}$.

ТеоремА ББВ (Ботт, Борель, Вейль, см. [42], [62]). Пусть $E=L^{\lambda}-$ неприводимый конечномерный $\mathfrak{g}$-модуль со старшим весом ${ }^{5)} \lambda$ такой, что $E \simeq E^{*}$. Тогда $H^{i}\left(\mathfrak{g}_{-} ; E\right)$ - прямая сумма $\mathfrak{l}$-модулей с младиими весами $-w_{i, j}(\lambda+\rho)+\rho$, где $w_{i, j} \in W(I)_{i}$, см. (19); любой такой модуль входит в сумму с кратностью 1.

При $i=2$ теорема ББВ описывает неголономные аналоги тензоров Вейля. Теорема, приведеная ниже в п. 5.4, описывает неголономные аналоги тензоров Римана. Чтобы доказать теорему пункта 5.4, нам потребуется следующая лемма.

5.3. Лемма. Пусть $E=L^{\lambda}$ - неприводимый (конечномерный) $\mathfrak{g}$-модуль со стариим весом $\lambda$ такой, что $E \simeq E^{*}$. Пусть $V$ есть p-инвариантное пространство в E, содержащее

$$
E_{-}=\bigoplus_{\left(\mu=\sum k_{i} \alpha_{i} \mid k_{i}<0\right)} E_{\mu} .
$$

Пусть любой вес $\mu$ модуля $E / V$ имеет вид $\mu=-\sum a_{i} \alpha_{i}$, где $a_{i} \geqslant 0$.

Тогда при любом $i<\operatorname{rkg}$ имеется изоморфизм $\mathfrak{l}$-модулей

$$
H^{i}\left(\mathfrak{g}_{-} ; V\right) \simeq H^{i}\left(\mathfrak{g}_{-} ; E\right) \oplus H^{i-1}\left(\mathfrak{g}_{-} ; E / V\right) .
$$

ДоКАЗАТЕЛЬСтво. Как хорошо известно [53], с каждой короткой точной последовательностью $\mathfrak{g}_{-}$-модулей

$$
0 \longrightarrow V \longrightarrow E \longrightarrow E / V \longrightarrow 0
$$

ассоциирована длинная точная последовательность

$$
\begin{aligned}
0 \longrightarrow & H^{0}\left(\mathfrak{g}_{-} ; V\right) \longrightarrow H^{0}\left(\mathfrak{g}_{-} ; E\right) \stackrel{\varphi_{0}}{\longrightarrow} H^{0}\left(\mathfrak{g}_{-} ; E / V\right) \longrightarrow \\
\longrightarrow & H^{1}\left(\mathfrak{g}_{-} ; V\right) \longrightarrow H^{1}\left(\mathfrak{g}_{-} ; E\right) \stackrel{\varphi_{1}}{\longrightarrow} H^{1}\left(\mathfrak{g}_{-} ; E / V\right) \longrightarrow \cdots \\
& \quad \cdots \longrightarrow H^{i}\left(\mathfrak{g}_{-} ; V\right) \longrightarrow H^{i}\left(\mathfrak{g}_{-} ; E\right) \stackrel{\varphi_{i}}{\longrightarrow} H^{i}\left(\mathfrak{g}_{-} ; E / V\right) \longrightarrow \cdots
\end{aligned}
$$

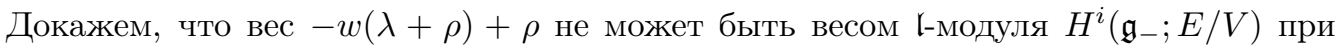
$i<\mathrm{rkg}$. Действительно, каждый вес пространства $H^{i}\left(\mathfrak{g}_{-} ; E / V\right)$, являющегося подфактором модуля $E^{i}\left(\mathfrak{g}_{-}^{*}\right) \otimes E / V$, имеет вид $\gamma_{1}+\cdots+\gamma_{k}+\mu$, где $\gamma_{1}, \ldots, \gamma_{k}$ - разные положительные корни, которые не принадлежат решетке $Q\left(B_{I}\right)$, а $\mu$ - вес модуля $E$ вида $\mu=\sum_{a_{i} \geqslant 0} a_{i} \alpha_{i}$.

5) Теорема ББВ была первоначально сформулирована без дополнительного требования $E \simeq E^{*}$. Мы налагаем его для простоты, однако во всех случаях, которые нас интересуют $(E=\mathfrak{g})$, оно выполнено. 
Предположим, что

$$
-w(\lambda+\rho)+\rho=\gamma_{1}+\cdots+\gamma_{k}+\mu
$$

Тогда $-\mu-w(\lambda)-w(\rho)+\rho=\gamma_{1}+\cdots+\gamma_{k}$. Положим

$$
R_{W}^{-}=\left\{a \in R_{+} \mid w^{-1}(\alpha)>0\right\}, \quad R_{W}^{+}=\left\{a \in R_{+} \mid w^{-1}(\alpha)<0\right\} .
$$

Пусть $\gamma_{1}, \ldots, \gamma_{s} \in R_{W}^{-}$и $\gamma_{s+1}, \ldots, \gamma_{k} \in R_{W}^{+}$.

Напомним, что

$$
\rho-w(\rho)=\sum_{\beta \in R_{W}^{-}} \beta .
$$

Поскольку $E=E^{*}$, вес $-\mu$ является весом модуля $E$; но тогда

$$
\begin{aligned}
w^{-1}\left(\gamma_{1}+\cdots+\gamma_{k}\right) & =\left(w^{-1}\left(\gamma_{1}\right)+\cdots+w^{-1}\left(\gamma_{s}\right)\right)+\left(w^{-1}\left(\gamma_{s+1}\right)+\cdots+w^{-1}\left(\gamma_{k}\right)\right)= \\
& =\left(-\lambda-w^{-1}(\mu)\right)+w^{-1}\left(\sum_{\beta \in R_{W}^{-}} \beta\right) .
\end{aligned}
$$

Из того, что $-w^{-1}(\mu)-$ вес, следует, что $\lambda+w^{-1}(\mu) \in Q_{+}(R)$. Поэтому

$$
-\left(\lambda+w^{-1}(\mu)\right)+w^{-1}\left(\sum_{\beta \in R_{W}^{-}} \beta\right) \in-Q_{+}(R) .
$$

С другой стороны,

$$
w^{-1}\left(\gamma_{s+1}\right)+\cdots+w^{-1}\left(\gamma_{k}\right) \in Q_{+}(R),
$$

и все веса $w^{-1}\left(\gamma_{i}\right)$ при $i \leqslant s$ входят в качестве слагаемых в выражение $\sum_{\beta \in R_{W}^{-}} w^{-1} \beta$ и, следовательно, взаимно уничтожаются.

Аналогично получаем, что

$$
\begin{aligned}
Q_{+}(R) & \ni w^{-1}\left(\gamma_{s+1}\right)+\cdots+w^{-1}\left(\gamma_{k}\right)= \\
& =-\left(\lambda+w^{-1}(\mu)\right)+w^{-1}\left(\sum_{\beta \in R_{W}^{-} \backslash\left\{\gamma_{1}, \ldots, \gamma_{s}\right\}} \beta\right) \in-Q_{+}(R) .
\end{aligned}
$$

Итак, $s=k$, а $\lambda=-w^{-1}(\mu)$. Другими словами,

$$
-\mu=w(\lambda)=-\sum a_{i} \alpha_{i} \quad \text { при } \quad a_{i} \geqslant 0 .
$$

Поскольку $\lambda$ - доминантный вес, то $\lambda=\sum m_{i} \alpha_{i}$, где все $m_{i} \in \mathbb{Q}$ положительны. Это верно для любого фундаментального веса, как следует из таблиц, приведенных в [63].

По предположению $l(w)<\mathrm{rkg}$, следовательно, $w=s_{\alpha_{1}} \ldots s_{\alpha_{r}}$, где $r<\mathrm{rk} \mathfrak{g}$. Положим $B_{w}:=\left\{\alpha_{i_{1}}, \ldots, \alpha_{i_{r}}\right\} \subset B$. Для любого $x \in Q(B) \otimes_{\mathbb{Z}} \mathbb{R}$ имеет место равенство $x-w(x)=-\sum_{\alpha \in B_{w}} n_{\alpha} \alpha$. Но тогда (напомним что $\varpi_{j}-$ это $j$-й фундаментальный 
вес [63]) $\left(x-w(x), \varpi_{j}\right)=0$ для некоторого $j \leqslant \operatorname{rkg}$. Поэтому $\left(\lambda, \varpi_{j}\right)=\left(w(\lambda), \varpi_{j}\right)$. Теперь заметим, что

$$
\left(\lambda, \varpi_{j}\right)=m_{j}\left(\alpha_{j}, \varpi_{j}\right)>0, \quad\left(w(\lambda), \varpi_{j}\right)=-a_{j}\left(\alpha_{j}, \varpi_{j}\right) \leqslant 0 .
$$

Это противоречие показывает, что $\varphi_{i}=0$ при $i<\mathrm{rkg}$, см. (20). Это в свою очередь означает, что при $i=1, \ldots, \mathrm{rk} \mathfrak{g}-1$ существуют короткие точные последовательности l-гомоморфизмов

$$
0 \longrightarrow H^{i-1}\left(\mathfrak{g}_{-} ; E / V\right) \longrightarrow H^{i}\left(\mathfrak{g}_{-} ; V\right) \longrightarrow H^{i}\left(\mathfrak{g}_{-} ; E\right) \longrightarrow 0 .
$$

5.4. Теорема. Имеет место следующее соотношение:

$$
H^{2}\left(\mathfrak{g}_{-} ; \mathfrak{g}_{-} \oplus \mathfrak{l}^{(1)}\right)=H^{2}\left(\mathfrak{g}_{-} ; \mathfrak{g}\right) \oplus H^{1}\left(\mathfrak{g}_{-} ;\left(\mathfrak{g}_{-} \oplus \mathfrak{z}\right)^{*}\right)
$$

ДокАЗАТЕЛЬСтво. Положим $E=\mathfrak{g}, V=\mathfrak{g}_{-} \oplus \mathfrak{l}^{(1)}$. По лемме 5.3

$$
H^{2}\left(\mathfrak{g}_{-} ; \mathfrak{g}_{-} \oplus \mathfrak{l}^{(1)}\right)=H^{2}\left(\mathfrak{g}_{-} ; \mathfrak{g}\right) \oplus H^{1}\left(\mathfrak{g}_{-} ; \mathfrak{g} /\left(\mathfrak{g}_{-} \oplus \mathfrak{l}^{(1)}\right)\right) .
$$

Осталось проверить, что $\mathfrak{g} /\left(\mathfrak{g}_{-} \oplus \mathfrak{l}^{(1)}\right)=\left(\mathfrak{g}_{-} \oplus \mathfrak{z}\right)^{*}$. Действительно, форма Киллинга $K$ устанавливает изоморфизм $\mathfrak{g}=\mathfrak{g}^{*}$, следовательно,

$$
\left(\mathfrak{g} /\left(\mathfrak{g}_{-} \oplus \mathfrak{l}^{(1)}\right)\right)^{*}=\left\{x \in \mathfrak{g} \mid K\left(x, \mathfrak{g}_{-} \oplus \mathfrak{l}^{(1)}\right)=0\right\}=\mathfrak{g}_{-} \oplus \mathfrak{z},
$$

где $\mathfrak{z}=\left\{z \in \mathfrak{l} \mid K\left(z, h_{\alpha}\right)=0\right.$ при $\left.\alpha \in B_{I}\right\}$, как и требовалось.

Заметим, что $\operatorname{dim} \mathfrak{z}$ равна мощности множества $I$, в частности, она равна 1 в разделах 6,8 и равна 2 в разделе 7.

СлеДСТВИЕ. Пусть $B_{1}=B \backslash B_{I}$, a $R_{1}$ - система корней, порожденная множеством $B_{1}$,

$$
\mathfrak{g}_{-}^{a b}=\left(\mathfrak{g}_{-} / \mathfrak{g}_{-}^{(1)}\right)^{*}=H^{1}\left(\mathfrak{g}_{-}\right) .
$$

1. Следующая последовательность является точной:

$$
\begin{aligned}
0 \longrightarrow \mathfrak{g}_{-}^{a b} \longrightarrow \mathfrak{z}^{*} \otimes \mathfrak{g}_{-}^{a b} \longrightarrow H^{1}\left(\mathfrak{g}_{-} ; \mathfrak{g} /\left(\mathfrak{g}_{-} \oplus \mathfrak{r}^{(1)}\right)\right) \longrightarrow H^{1}\left(\mathfrak{g}_{-} ; \mathfrak{g}_{-}^{*}\right) \longrightarrow \\
\longrightarrow H^{2}\left(\mathfrak{g}_{-}\right) \oplus \bigoplus_{w \in W\left(R_{1}\right)_{(2)}} L^{\rho-w(\rho)} \longrightarrow 0 .
\end{aligned}
$$

2. Если $\operatorname{dim} \mathfrak{z}=1$, то последовательность

$$
0 \longrightarrow H^{1}\left(\mathfrak{g}_{-} ;\left(\mathfrak{g}_{-} \oplus \mathfrak{z}\right)^{*}\right) \longrightarrow H^{1}\left(\mathfrak{g}_{-} ; \mathfrak{g}_{-}^{*}\right) \longrightarrow H^{2}\left(\mathfrak{g}_{-}\right) \longrightarrow 0
$$

является точной. В частности, если $\mathfrak{g}_{-}$- алгебра Гейзенберга (в случае контактной градуировки), то

$$
H^{2}\left(\mathfrak{g}_{-} ; \mathfrak{g}_{-} \oplus \mathfrak{l}^{(1)}\right) \simeq H^{2}\left(\mathfrak{g}_{-} ; \mathfrak{g}\right) \oplus S^{2}\left(\mathfrak{g}_{-} / \mathfrak{z}\left(\mathfrak{g}_{-}\right)\right)^{*}=H^{2}\left(\mathfrak{g}_{-} ; \mathfrak{g}\right) \oplus S^{2}\left(\mathfrak{g}_{-1}^{*}\right)
$$

3. Если $\mathfrak{g}_{-}=\mathfrak{g}_{-1}($ абелева алгебра Ли), то

$$
H^{2}\left(\mathfrak{g}_{-} ; \mathfrak{g}_{-} \oplus \mathfrak{l}^{(1)}\right) \simeq H^{2}\left(\mathfrak{g}_{-} ; \mathfrak{g}\right) \oplus S^{2}\left(\mathfrak{g}_{-}^{*}\right)=H^{2}\left(\mathfrak{g}_{-} ; \mathfrak{g}\right) \oplus S^{2}\left(\mathfrak{g}_{-1}^{*}\right) .
$$


ДокАЗАТЕЛЬСТво. Из короткой точной последовательности

$$
0 \longrightarrow\left(\mathfrak{g}_{-} \oplus \mathfrak{l}\right) /\left(\mathfrak{g}_{-} \oplus \mathfrak{l}^{(1)}\right) \longrightarrow \mathfrak{g} /\left(\mathfrak{g}_{-} \oplus \mathfrak{l}^{(1)}\right) \longrightarrow \mathfrak{g} /\left(\mathfrak{g}_{-} \oplus \mathfrak{l}\right) \longrightarrow 0
$$

где $\mathfrak{g} /\left(\mathfrak{g}_{-} \oplus \mathfrak{l}\right)=\mathfrak{g}_{-}^{*}$ и $\left(\mathfrak{g}_{-} \oplus \mathfrak{l}\right) /\left(\mathfrak{g}_{-} \oplus \mathfrak{l}^{(1)}\right)=\mathfrak{z}^{*}$, мы выводим длинную точную последовательность

$$
\begin{aligned}
0 \longrightarrow \mathfrak{g}_{-}^{a b} \longrightarrow \mathfrak{z}^{*} \otimes H^{1}\left(\mathfrak{g}_{-}\right) \longrightarrow H^{1}\left(\mathfrak{g}_{-} ; \mathfrak{g} /\left(\mathfrak{g}_{-} \oplus \mathfrak{l}^{(1)}\right)\right) \longrightarrow \\
\left.\longrightarrow H^{1}\left(\mathfrak{g}_{-} ; \mathfrak{g}_{-}^{*}\right) \stackrel{\psi}{\longrightarrow} H^{2}\left(\mathfrak{g}_{-} ; \mathfrak{z}^{*}\right)=H^{2}\left(\mathfrak{g}_{-}\right) \otimes \mathfrak{z}^{*}\right) \longrightarrow \cdots
\end{aligned}
$$

Чтобы вычислить образ отображения $\psi$, рассмотрим l-модуль

$$
\begin{aligned}
M=\left\{f \in \operatorname{Hom}\left(\mathfrak{g}_{-}, \mathfrak{g} /\left(\mathfrak{g}_{-} \oplus \mathfrak{l}^{(1)}\right)\right) \mid X f(Y)-Y f(X)-f([X, Y]) \in \mathfrak{z}^{*}\right. \\
\text { для любых } \left.X, Y \in \mathfrak{g}_{-}\right\} .
\end{aligned}
$$

Из общих свойств длинных точных последовательностей [53] мы выводим, что

$$
\operatorname{Im}(\psi)=M / Z^{1}\left(\mathfrak{g}_{-} ; \mathfrak{g} /\left(\mathfrak{g}_{-} \oplus \mathfrak{l}^{(1)}\right)\right) .
$$

По теореме ББВ

$$
H^{2}\left(\mathfrak{g}_{-}\right)=\bigoplus_{\left\{w \mid l(w)=2, w\left(B \backslash B_{I}\right)>0\right\}} L^{w(\rho)-\rho} .
$$

Поскольку $M$ - прямая сумма своих весовых подпространств относительно $\mathfrak{h}$, изучим подпространства $M_{\rho-w(\rho)}$. Вес элемента $f \in M$ равен $w(\rho)-\rho$ тогда и только тогда, когда $f$ - линейное отображение из пространства $\mathfrak{n}_{-\gamma}$ в пространство $\left(\mathfrak{g} /\left(\mathfrak{g}_{-} \oplus \mathfrak{l}^{(1)}\right)\right)_{\rho-w(\rho)-\gamma}$. Поэтому либо $\rho-w(\rho)-\gamma=\gamma^{\prime} \in R$, либо $\rho-w(\rho)-\gamma=0$.

Вторую возможность мы отбрасываем, поскольку $\rho-w(\rho)-$ не корень. Действительно, $l(w)=2 \neq 1$.

В первом случае $\rho-w(\rho)=\gamma+\gamma^{\prime}$, где $\gamma, \gamma^{\prime} \in R_{+}$. Если $\gamma=\gamma^{\prime}$, то $\rho-w(\rho)=2 \gamma$, откуда следует, что $w^{-1}(\gamma)<0$. Но $\operatorname{card}\left(R_{W}^{-}\right)=2$ и $\rho-w(\rho)=2 \gamma=\gamma_{1}+\gamma_{2}$, где $\gamma_{1}, \gamma_{2} \in R_{W}^{-}$. Поскольку один из корней $\gamma_{i}$ равен $\gamma$, то и другой равен $\gamma$. Это противоречит предположению $\gamma_{1} \neq \gamma_{2}$.

Итак, $\rho-w(\rho)=\gamma+\gamma^{\prime}$, где $\gamma \neq \gamma^{\prime}$. Нетрудно вывести отсюда, что $w^{-1}(\gamma)<0$ и $w^{-1}\left(\gamma^{\prime}\right)<0$. Следовательно, $f\left(\mathfrak{n}_{-\delta}\right) \neq 0$, только если $w^{-1}(\delta)<0$, где $f \in M_{\rho-w(\rho)}$. В этом случае $f\left(\mathfrak{n}_{-\delta}\right) \in\left(\mathfrak{g} /\left(\mathfrak{g}_{-} \oplus \mathfrak{l}^{(1)}\right)\right)_{\delta^{\prime}}$, где $w^{-1}\left(\delta^{\prime}\right)<0$. Кроме того, поскольку $\delta, \delta^{\prime} \notin R$, то $\left[X_{-\delta}, X_{-\delta^{\prime}}\right]=0$ для корневых векторов $X_{-\delta}, X_{-\delta^{\prime}}$ (в противном случае $\delta, \delta^{\prime} \in R_{W}^{-}$и $\left.l(w)>2\right)$.

С другой стороны, любое отображение $f_{w}: \mathfrak{n}_{-\delta} \rightarrow \mathfrak{g} /\left(\mathfrak{g}_{-} \oplus \mathfrak{l}^{(1)}\right)$, обращающееся в нуль вне подпространства $\mathfrak{n}_{-\gamma} \oplus \mathfrak{n}_{-\gamma^{\prime}}$, где $\left\{\gamma, \gamma^{\prime}\right\}=R_{W}^{-}$, и такое, что вес элементов $f_{w}\left(\mathfrak{n}_{-\gamma}\right)$ равен $\gamma^{\prime}$, в то время как вес элементов $f_{w}\left(\mathfrak{n}_{-\gamma^{\prime}}\right)$ равен $\gamma$, принадлежит, очевидно, пространству $M_{\rho-w(\rho)}$. Это значит, что $\operatorname{dim} M_{\rho-w(\rho)} \leqslant 2$.

Пусть $w=s_{\alpha_{1}} s_{\alpha_{2}}$, где $\alpha_{2} \in B_{1}$ и $\left(\alpha_{1}, \alpha_{2}\right) \neq 0$. Тогда $\operatorname{dim} M_{\rho-w(\rho)}=1$, как показывает следующее вычисление. Пусть

$$
f\left(X_{-\alpha_{2}}\right)=c X_{\alpha_{1}+\alpha_{2}}^{\prime}, \quad f\left(X_{-\left(\alpha_{1}+\alpha_{2}\right)}\right)=c^{\prime} X_{\alpha_{2}}^{\prime},
$$


где векторы со штрихом принадлежат факторпространству. Тогда для подходящих коэффициентов $c$ и $c^{\prime}$ получаем, что

$$
\begin{aligned}
& X_{-\left(\alpha_{1}+\alpha_{2}\right)} f\left(X_{-\alpha_{2}}\right)-X_{-\alpha_{2}} f\left(X_{-\left(\alpha_{1}+\alpha_{2}\right)}^{\prime}\right)= \\
& \quad=-c H_{\alpha_{1}+\alpha_{2}}^{\prime}+c^{\prime} H_{\alpha_{2}}^{\prime} \in \mathbb{C} H_{\alpha_{1}}^{\prime} \subset\left(\mathfrak{g}_{-} \oplus \mathfrak{l}\right) /\left(\mathfrak{g}_{-} \oplus \mathfrak{l}^{(1)}\right) .
\end{aligned}
$$

Если $w=s_{\alpha_{1}} s_{\alpha_{2}}$, где $\alpha_{1}, \alpha_{2} \in B_{I}$, то $\operatorname{dim} M_{\rho-w(\rho)}=2$. Для любого такого $w$ имеем

$$
M_{\rho-w(\rho)} \cap Z^{2}\left(\mathfrak{g}_{-} ; \mathfrak{g} /\left(\mathfrak{g}_{-} \oplus \mathfrak{l}^{(1)}\right)\right) \neq 0,
$$

откуда следует утверждение пункта 1.

Общее утверждение пункта 2 доказывается непосредственно. Его частный случай вытекает из очевидных равенств $H^{1}\left(\mathfrak{g}_{-} ; \mathfrak{g}_{-}^{*}\right)=\mathfrak{g}_{-}^{*} \otimes \mathfrak{g}_{-}^{*}$ и $H^{2}\left(\mathfrak{g}_{-}\right)=E^{2}\left(\mathfrak{g}_{-}^{*}\right)$.

Для доказательства общего утверждения пункта 2 положим $\mathfrak{g}_{-}^{\prime}=\mathfrak{g}_{-} / \mathfrak{z}\left(\mathfrak{g}_{-}\right)$. Ясно, что $\mathfrak{g}_{-}^{\prime}$ - тривиальный $\mathfrak{g}_{-}^{\prime}$-модуль. Следовательно, последовательность

$$
0 \longrightarrow \mathfrak{g}_{-}^{\prime} \longrightarrow \mathfrak{g}_{-}^{\prime} \longrightarrow \mathbb{C} \longrightarrow 0
$$

точна, а с ней точна и последовательность

$$
0 \longrightarrow \mathbb{C} \longrightarrow \mathfrak{g}_{-}^{\prime} \otimes H^{1}\left(\mathfrak{g}_{-}\right) \longrightarrow H^{1}\left(\mathfrak{g}_{-} ; \mathfrak{g}_{-}^{\prime}\right) \stackrel{f}{\longrightarrow} H^{2}\left(\mathfrak{g}_{-}\right) \longrightarrow \ldots
$$

Нетрудно заметить, что образ любого коцикла $\mathfrak{g}_{-} \longrightarrow \mathfrak{g}_{-}^{\prime}$ под действием отображения $f$ принадлежит $E^{2}\left(\mathfrak{g}_{-}^{\prime}\right)$, следовательно, $f=0$. Но тогда пространство $H^{1}\left(\mathfrak{g}_{-} ; \mathfrak{g}_{-}^{\prime}\right)$ изоморфно подпространству $\left(\mathfrak{g}_{-}^{\prime} \otimes \mathfrak{g}_{-}^{\prime}\right)_{0}$ бесследовых операторов из пространства $\mathfrak{g}_{-}^{\prime} \otimes \mathfrak{g}_{-}^{\prime}$. Осталось заметить, что $H^{2}\left(\mathfrak{g}_{-}\right) \oplus \mathbb{C}=E^{2}\left(\mathfrak{g}_{-}^{\prime}\right)$.

5.5. Число $\mathfrak{g}_{0}$-модулей. Следующая теорема помогает проверить результат применения теорем 5.2 и 5.4. Пусть $I R$ - число неприводимых компонент в $\mathfrak{g}_{0}$-модуле $H^{2}\left(\mathfrak{g}_{-} ;\left(\mathfrak{g}_{-}, \mathfrak{g}_{0}\right)_{*}\right)$.

Теорема. Имеет место следующее равенство: $I R=\frac{1}{2} c(c+1)+\sum c_{i}$.

ДокАЗАтЕЛЬСтво. Пусть $I R=\operatorname{card} W(I)_{2}$. Перечислим элементы длины 2 из $W(I)$. Ясно, что

$$
W\left(R_{I}\right)_{2}=\left\{w \in W\left(R_{I}\right) \mid l(w)=2\right\} \subset W(I)_{2} .
$$

Если $s_{i} s_{j} \in W(I)_{2} \backslash W\left(R_{I}\right)_{2}$, то $s_{j} \in B_{I}$ и $s_{i} \notin B_{I}$ (если $s_{j} \notin B_{I}$, то $s_{i} s_{j}\left(a_{j}\right)=$ $-s_{i}\left(a_{j}\right)<0$, что неверно).

Более того, $\left(\alpha_{i}, \alpha_{j}\right) \neq 0$, поскольку в противном случае $s_{i} s_{j}\left(\alpha_{i}\right)=-\alpha_{i}<0$.

Пусть $\operatorname{rk} R=r$. Пусть $R=R_{1} \amalg \cdots \coprod R_{s}$ - представление системы $R$ в качестве объединения связанных компонент. Пусть $B=\left\{\alpha_{1}, \ldots, \alpha_{r}\right\}$ - система простых корней системы $R$. Тогда

$$
\begin{aligned}
& W(R)_{2}=\left\{s_{i} s_{j} \quad \text { при } \quad i \neq j\right\}= \\
& =\left\{s_{i} s_{j} \quad \text { при } \quad i<j\right\} \coprod\left\{s_{i} s_{j} \quad \text { при } \quad i>j \mid\left(\alpha_{i}, \alpha_{j}\right) \neq 0\right\} .
\end{aligned}
$$

3 Теоретическая и математическая физика, т. 153, № 2, 2007 г. 
Следовательно

$$
\operatorname{card} W(R)_{2}=\frac{c(c-1)}{2}+\operatorname{card}(E)=\frac{c(c-1)}{2}+c-s
$$

(здесь $\operatorname{card}(E)$ - количество ребер графа Дынкина системы $R$, которые подсчитываются без учета кратности). Если $\alpha \in R \backslash R_{I}$ и $\left(\alpha, R_{I}^{(i)}\right) \neq 0$ при некотором $i$, то существует единственный вес $\beta \in R_{I}^{(i)}$ такой, что $(\alpha, \beta) \neq 0$. Действительно, если таких корней два (скажем, $\beta_{1}$ и $\beta_{2}$ ), то граф Дынкина содержит цикл

$$
\alpha-\beta_{1}-\gamma_{1}-\gamma_{2}-\ldots-\gamma_{s}-\beta_{2}-\alpha .
$$

Действительно, граф $D_{I}^{(i)}$ связен и $\alpha \notin R_{I}^{(i)}$, следовательно, $\gamma_{1}, \ldots, \gamma_{s} \in D_{I}^{(i)}$.

Все это показывает, что

$$
\begin{aligned}
\operatorname{card}\left(W_{I}\right)_{2} & =\operatorname{card}\left(W\left(R_{I}\right)\right)_{2}+\sum_{i=1}^{s}\left(c_{i}+1\right)= \\
& =\frac{c(c-1)}{2}+c-s+\left(\sum_{i=1}^{s} c_{i}\right)+s=\frac{c(c+1)}{2}+\sum_{i=1}^{s} c_{i} .
\end{aligned}
$$

\section{6. ЯВНЫЕ РЕЗУЛЬТАТЫ: ПРОСТЕЙШИЕ ФЛАГИ}

Мы рассмотрим стандартную нумерацию вершин графа Дынкина (такую же, как в [63] или [64]). Пусть $k_{i}$ - коэффициент при $i$-м простом корне в разложении старшего корня по простым корням. Для нильпотентной подалгебры $\mathfrak{g}_{-}=\bigoplus_{-d \leqslant j \leqslant-1} \mathfrak{g}_{j}$, дополнительной к максимальной параболической подалгебре $\mathfrak{p}_{i}$ (соответствующей выбору $i$-го простого кокорня), глубина $d$ градуировки равна $k_{i}$. Вершины, помеченные числами $k_{i}=1$, соответствуют эрмитовым симметрическим пространствам, рассмотренным в работах [32] для конформного случая и в работе [43] для риманова случая, поэтому в таблицах 1-4 мы не приводим соответствующие грассманианам случаи алгебры Ли $\mathfrak{s l}$ с одной избранной вершиной графа Дынкина. Для оставшихся случаев классических областей $X=G / P$ мы представляем младшие веса неголономных тензоров Вейля (неголономных только при $k_{i} \neq 1$ ) относительно факторгруппы Леви, соответствующей такой $\mathbb{Z}$-градуировке алгебры $\mathfrak{g}$, при которой избранный кокорень (вершина) имеет степень -1 , тогда как все остальные кокорни имеют степень 0.

Веса простых корней задаются столбцами матрицы Картана. Положим

$$
H^{1}:=H^{1}\left(\mathfrak{g}_{-} ;\left(\mathfrak{g}_{-} \oplus \mathfrak{z}\right)^{*}\right), \quad H^{2}:=H^{2}\left(\mathfrak{g}_{-} ; \mathfrak{g}\right) .
$$

В таблицах 1-4 представлены младшие веса неприводимых l-компонент пространства $H^{2}$ в терминах базиса из простых корней (столбцов матрицы Картана СМ), поскольку в этом случае их легче сосчитать, и в терминах базиса из фундаментальных весов $(F W)$, поскольку в таком виде они привычнее ${ }^{6)}$.

\footnotetext{
6) Программа SuperLie пересчитывает FW-веса в CM-веса и обратно за мгновение.
} 
Старшие веса неприводимых l-компонент пространства $H^{1}$ представлены только в терминах фундаментальных весов. Для экономии места столбец $H^{1}$ содержит все неприводимые l-модули, которые мы нашли, кроме одного: для каждой алгебры с $i$-й выделенной вершиной, всегда существует компонента со старшим весом вида $(\underbrace{0, \ldots, 0,2}_{i}, 0, \ldots, 0)$.

Мы задаем веса $\widehat{\mathfrak{g}_{0}}=\mathfrak{l}^{(1)}$-модулей, образующих неконформную часть неголономного тензора Римана, относительно той же картановской подалгебры $\mathfrak{g}_{0}=\mathfrak{l}$, что мы использовали при описании неголономных тензоров Вейля.

ЗАмечАния. 1. Порядки почти всех структурных функций равны двум. Исключения составляют один случай порядка 4 для алгебры Ли $\mathfrak{g}(2)$ и несколько случаев порядка 3 для алгебр Ли $\mathfrak{f}(4)$ и $\mathfrak{o}(2 n+1)$.

2. Положительные координаты младших весов не являются ошибками: при задании в терминах матрицы Картана СМ они появляются только в местах, соответствующих центру алгебры Ли l; остальные (неположительные) координаты соответствуют полупростой части алгебры $\mathfrak{l}$.

\section{7. ЯВНЫЕ РЕЗУЛЬТАТЫ: СЛУЧАЙ 3 ТЕОРЕМЫ ЯМАГУЧИ}

В случае 3 теоремы Ямагучи (для полноты мы рассматриваем также случай алгебры Ли $\mathfrak{s l}$ с избранным последним простым кокорнем) младшие веса $\mathfrak{g}_{0}$-модуля $H^{2}\left(\mathfrak{g}_{-} ; \mathfrak{g}\right)$ и старшие веса модуля $H^{1}\left(\mathfrak{g}_{-} ;\left(\mathfrak{g}_{-} \oplus \mathfrak{z}\right)^{*}\right)$, а также их степени перечислены ниже в этом разделе, кроме того, приведены коциклы, имеющие простой вид.

А. Случай $\mathfrak{g}=\mathfrak{s l}(n+1)$ (веса представлены относительно стандартных образующих картановской подалгебры алгебры Ли $\mathfrak{g}$, т.е. относительно $e_{1}^{1}-e_{2}^{2}, e_{2}^{2}-e_{3}^{3}, \ldots$, $\left.e_{n}^{n}-e_{n+1}^{n+1}\right)$.

Избранные простые кокорни $(1,2)$ :

1) $\operatorname{deg}=0$ для веса $(-3,3,0,-1,0, \ldots, 0,-1)$;

2) $\mathrm{deg}=2$ для веса $(4,-1,-1,0, \ldots, 0,-1)$;

$3) \mathrm{deg}=3$ для веса $(0,4,-3,0, \ldots, 0,-1)$.

Избранные простые кокорни $(1, i)$, где $3 \leqslant i \leqslant n-2$ :

1) $\operatorname{deg}=0$ для веса $(-1,0, \ldots, 0,-1)+(\ldots, 0,-1,0,-3,-2,0, \ldots)$;

2) $\operatorname{deg}=0$ для веса $(-1,0, \ldots, 0,-1)+(\ldots, 0,-2,-3,0,-1,0, \ldots)$;

$3) \mathrm{deg}=1$ для веса $(3,-2,0, \ldots, 0,-1)+(\ldots, 0,-1,2,-1,0, \ldots)$;

4) $\operatorname{deg}=1$ для веса $(4,-1,-1,0,0,0,-1)$.

Для большей наглядности веса представлены в пунктах $1,2,3$ в виде суммы постоянной части и части, зависящей от $i$ (на $i$-м месте стоит $-3,-3$ и 2 , соответственно).

Избранные простые кокорни $(1, n-1)$ (один вес отличается от вышеприведенного случая):

1) $\operatorname{deg}=0$ для веса $(-1,0, \ldots, 0,-1,0,3,-3)$ (как и выше),

2) $\mathrm{deg}=1$ для веса $(-1,0, \ldots, 0,-3,4,0)$,

$3) \operatorname{deg}=1$ для веса $(3,-2,0, \ldots, 0,-1,2,-2)$ (как и выше), 
4) $\operatorname{deg}=1$ для веса $(4,-1,-1,0, \ldots, 0,-1)$ (как и выше).

Исключительные случаи:

случай $\mathfrak{s l}(5)$, избранные простые кокорни $(1,2)$ :

1) $\operatorname{deg}=0$ для веса $(-3,3,0,-2)$,

2) $\operatorname{deg}=2$ для веса $(4,-1,-1,-1)$,

ТАБлицА 1. Неголономные тензоры для флагов, ассоциированных с исключительными простыми группами Ли

\begin{tabular}{|c|c|c|c|c|c|}
\hline $\mathfrak{g}$ & Вершина & $k_{i}$ & $\mathrm{CM}$ & FW & $H^{1}$ \\
\hline \multirow[t]{2}{*}{$\mathfrak{g}(2)$} & 1 & 3 & $(8,-4)$ & $(4,0)$ & $(4,2)$ \\
\hline & 2 & 2 & $(-7,4)$ & $(-2,1)$ & $(2,2)$ \\
\hline \multirow[t]{4}{*}{$\mathfrak{f}(4)$} & 1 & 2 & $(3,0,-1,-1)$ & $(0,-3,-3,-2)$ & $(2,3,2,1)$ \\
\hline & 2 & 4 & $\begin{array}{l}(0,3,-2,-1) \\
(-3,4,-1,-2)\end{array}$ & $\begin{array}{c}(-1,-2,-3,-2) \\
(-2,-1,2,-2)\end{array}$ & $\begin{array}{l}(0,3,2,1) \\
(1,2,1,0)\end{array}$ \\
\hline & 3 & 3 & $\begin{array}{c}(0,-6,4,0) \\
(-1,-2,3,-3)\end{array}$ & $\begin{array}{c}(-2,-4,0,0) \\
(-2,-3,-1,-2)\end{array}$ & $\begin{array}{l}(0,2,2,0) \\
(0,1,2,0)\end{array}$ \\
\hline & 4 & 2 & $(0,-2,-1,4)$ & $(-2,-4,-2,1)$ & $(0,2,2,2)$ \\
\hline \multirow[t]{6}{*}{$\mathfrak{e}(6)$} & 1 & 1 & $(3,0,-1,0,0,-1)$ & $(1,-1,-3,-2,-1,-2)$ & $(2,2,2,1,0,1)$ \\
\hline & 2 & 2 & $\begin{array}{c}(0,3,-2,0,0,-1) \\
(-2,3,0,-1,0,-2)\end{array}$ & $\begin{array}{c}(0,0,-3,-2,-1,-2) \\
(-1,0,-2,-2,-1,-2)\end{array}$ & $\begin{array}{l}(1,2,1,0,0,0) \\
(0,2,2,1,0,1)\end{array}$ \\
\hline & 3 & 3 & $\begin{array}{c}(0,-3,4,-3,0,0) \\
(0,-2,3,0,-1,-3) \\
(-1,0,3,-2,0,-3)\end{array}$ & $\begin{array}{c}(-1,-2,0,-2,-1,0) \\
(-1,-2,-1,-1,-1,-2) \\
(-1,-1,-1,-2,-1,-2)\end{array}$ & $\begin{array}{l}(0,1,2,1,0,0) \\
(0,0,2,1,0,1) \\
(0,1,2,0,0,1)\end{array}$ \\
\hline & 4 & 2 & $\begin{array}{c}(0,0,-2,3,0,-1) \\
(0,-1,0,3,-2,-2)\end{array}$ & $\begin{array}{c}(-1,-2,-3,0,0,-2) \\
(-1,-2,-2,0,-1,-2)\end{array}$ & $\begin{array}{l}(0,0,1,2,1,0) \\
(0,1,2,2,0,1)\end{array}$ \\
\hline & 5 & 1 & $(0,0,-1,0,3,-1)$ & $(-1,-2,-3,-1,1,-2)$ & $(0,1,2,2,2,1)$ \\
\hline & 6 & 2 & $(0,-1,-1,-1,0,4)$ & $(-1,-2,-2,-2,-1,1)$ & $(0,1,2,1,0,2)$ \\
\hline \multirow[t]{7}{*}{$\mathfrak{e}(7)$} & 1 & 1 & $(3,0,-1,0,0,-1,0)$ & $(1,-1,-3,-4,-3,-2,-2)$ & $(2,2,2,2,1,0,1)$ \\
\hline & 2 & 2 & $\begin{array}{c}(0,3,-2,0,0,-1,0) \\
(-2,3,0,-1,0,-1,0)\end{array}$ & $\begin{array}{c}(0,0,-3,-4,-3,-2,-2) \\
(-1,0,-2,-4,-3,-2,-2)\end{array}$ & $\begin{array}{l}(1,2,1,0,0,0,0) \\
(0,2,2,2,1,0,1)\end{array}$ \\
\hline & 3 & 3 & $\begin{array}{c}(0,-2,3,0,-1,-1,-1) \\
(-1,0,3,-2,0,-1,0)\end{array}$ & $\begin{array}{l}(-1,-2,-1,-3,-3,-2,-2) \\
(-1,-1,-1,-4,-3,-2,-2)\end{array}$ & $\begin{array}{l}(0,1,2,1,0,0,0) \\
(0,0,2,2,1,0,1)\end{array}$ \\
\hline & 4 & 4 & $\begin{array}{c}(0,0,-2,3,-2,-1,0) \\
(0,0,-2,3,0,-2,-2) \\
(0,-1,0,3,-2,-1,-2)\end{array}$ & $\begin{array}{l}(-1,-2,-3,-2,-3,-2,-1) \\
(-1,-2,-3,-2,-2,-2,-2) \\
(-1,-2,-2,-2,-3,-2,-2)\end{array}$ & $\begin{array}{l}(0,0,1,2,1,0,0) \\
(0,0,0,2,1,0,1) \\
(0,0,1,2,0,0,1)\end{array}$ \\
\hline & 5 & 3 & $\begin{array}{c}(0,0,0,-3,4,0,0) \\
(0,0,-1,0,3,-3,-1)\end{array}$ & $\begin{array}{c}(-1,-2,-3,-4,0,0,-2) \\
(-1,-2,-3,-3,-1,-2,-2)\end{array}$ & $\begin{array}{l}(0,0,0,1,2,1,0) \\
(0,0,1,2,2,0,1)\end{array}$ \\
\hline & 6 & 2 & $(0,0,0,-1,-1,4,0)$ & $(-1,-2,-3,-4,-2,1,-2)$ & $(0,0,1,2,2,2,1)$ \\
\hline & 7 & 2 & $(0,0,-1,0,-1,-1,3)$ & $(-1,-2,-3,-3,-3,-2,0)$ & $(0,0,1,2,1,0,2)$ \\
\hline \multirow[t]{8}{*}{$\mathfrak{e}(8)$} & 1 & 2 & $(4,-1,-1,0,0,0,0,0)$ & $(-1,-2,-4,-5,-6,-4,-2,-3)$ & $(2,2,2,2,2,1,0,1)$ \\
\hline & 2 & 3 & $\begin{array}{c}(0,4,-3,0,0,0,0,0) \\
(-3,3,0,-1,0,0,0,0)\end{array}$ & $\begin{array}{c}(0,0,-4,-5,-6,-4,-2,-3) \\
(-2,-1,-3,-5,-6,-4,-2,-3)\end{array}$ & $\begin{array}{l}(1,2,1,0,0,0,0,0) \\
(0,2,2,2,2,1,0,1)\end{array}$ \\
\hline & 3 & 4 & $\begin{array}{c}(-1,-2,3,0,-1,0,0,0) \\
(-2,0,3,-2,0,0,0,0)\end{array}$ & $\begin{array}{l}(-2,-3,-2,-4,-6,-4,-2,-3) \\
(-2,-2,-2,-5,-6,-4,-2,-3)\end{array}$ & $\begin{array}{l}(1,2,1,0,0,0,0,0) \\
(0,2,2,2,2,1,0,1)\end{array}$ \\
\hline & 4 & 5 & $\begin{array}{c}(-1,0,-2,3,0,-1,0,-1) \\
(-1,-1,0,3,-2,0,0,0)\end{array}$ & $\begin{array}{l}(-2,-3,-4,-3,-5,-4,-2,-3) \\
(-2,-3,-3,-3,-6,-4,-2,-3)\end{array}$ & $\begin{array}{l}(0,0,1,2,1,0,0,0) \\
(0,0,0,2,2,1,0,1)\end{array}$ \\
\hline & 5 & 6 & $\begin{array}{c}(-1,0,0,-2,3,-2,0,0) \\
(-1,0,0,-2,3,0,-1,-2) \\
(-1,0,-1,0,3,-2,0,-2)\end{array}$ & $\begin{array}{l}(-2,-3,-4,-5,-4,-4,-2,-2) \\
(-2,-3,-4,-5,-4,-3,-2,-3) \\
(-2,-3,-4,-4,-4,-4,-2,-2)\end{array}$ & $\begin{array}{l}(0,0,0,1,2,1,0,0) \\
(0,0,0,0,2,1,0,1) \\
(0,0,0,1,2,0,0,1)\end{array}$ \\
\hline & 6 & 4 & $\begin{array}{c}(-1,0,0,0,-2,3,0,0) \\
(-1,0,0,-1,0,3,-2,-1)\end{array}$ & $\begin{array}{c}(-2,-3,-4,-5,-6,-3,0,-3) \\
(-2,-3,-4,-5,-5,-4,-2,-1)\end{array}$ & $\begin{array}{l}(0,0,0,0,1,2,1,0) \\
(0,0,0,1,2,2,0,1)\end{array}$ \\
\hline & 7 & 2 & $(-1,0,0,0,0,-1,2,0)$ & $(-1,0,0,0,-1,0,3,0)$ & $(0,0,0,1,2,2,2,1)$ \\
\hline & 8 & 3 & $(-1,0,0,0,-1,0,0,2)$ & $(-1,0,0,-1,0,-1,0,3)$ & $(0,0,0,1,2,1,0,2)$ \\
\hline
\end{tabular}


ТАБлицА 2. Неголономные тензоры для флагов, ассоциированных с $\mathfrak{o}(2 n)$

\begin{tabular}{|c|c|c|c|c|}
\hline Вершина & $k(i)$ & $r k$ & Bec & $H^{1}$ \\
\hline \multirow[t]{2}{*}{1} & \multirow[t]{2}{*}{1} & 4 & $(2,0,-1,-1)$ & $(2211)$ \\
\hline & & $4+l$ & $(2,0, \underbrace{-2, \ldots,-2},-1,-1)$ & $(\underbrace{2 \ldots 2}_{l-2}, 11)$ \\
\hline 2 & 2 & $5+l$ & $\begin{array}{c}(0,1,-2, \underbrace{-2, \ldots,-2}_{l},-1,-1) \\
(-1,1,-1, \underbrace{-2, \ldots,-2}_{l},-1,-1)\end{array}$ & $\begin{array}{l}(02211) \\
(12100)\end{array}$ \\
\hline 3 & 2 & $6+l$ & $\begin{array}{l}(-1,-2,0,1, \underbrace{-2, \ldots,-2}_{l},-1,-1) \\
-1,0,1,-2, \underbrace{-2, \ldots,-2}_{l},-1,-1)\end{array}$ & $\begin{array}{l}(0 \underbrace{2 \ldots 2}_{l-3} 11) \\
(121 \underbrace{0 \ldots 0}_{l-3})\end{array}$ \\
\hline \multirow[t]{2}{*}{$k<r k-6$} & \multirow[t]{2}{*}{2} & 7 & $\begin{array}{l}(-1, \underbrace{-2, \ldots,-2}_{k}-2,0,-1,-1,-1) \\
(-1, \underbrace{-2, \ldots,-2}_{k},-1,0,-2,-1,-1)\end{array}$ & $\begin{array}{l}(002211) \\
(012100)\end{array}$ \\
\hline & & $>7$ & $\begin{array}{l}(-1, \underbrace{-2, \ldots,-2}_{k}-2,0,-1, \underbrace{-2, \ldots,-2}_{l},-1,-1) \\
(-1, \underbrace{-2, \ldots,-2}_{k},-1,0,-2, \underbrace{-2, \ldots,-2}_{l},-1,-1)\end{array}$ & $\begin{array}{l}(0 \ldots 02211) \\
(0 \ldots 012100)\end{array}$ \\
\hline$\ldots$ & $\ldots$ & $\ldots$ & $\ldots$ & $\ldots$ \\
\hline \multirow[t]{3}{*}{ вилка } & \multirow[t]{3}{*}{2} & 4 & $\begin{array}{l}(0,1,-1,-1) \\
(-1,1,-1,0) \\
(-1,1,0,-1)\end{array}$ & $\begin{array}{l}(0211) \\
(1201) \\
(1210)\end{array}$ \\
\hline & & 5 & $\begin{array}{l}(-1,-2,0,-1,0) \\
(-1,-2,0,0,-1) \\
(-1,0,1,-1,-1)\end{array}$ & $\begin{array}{l}(00211) \\
(01201) \\
(01210)\end{array}$ \\
\hline & & $\begin{array}{c}\quad 5+k \\
k<r k-5\end{array}$ & $\begin{array}{l}(-1, \underbrace{-2, \ldots,-2}_{k},-2,0,-1,0) \\
(-1, \underbrace{-2, \ldots,-2}_{k},-2,0,0,-1) \\
(-1, \underbrace{-2, \ldots,-2}_{k},-1,0,-1,-1)\end{array}$ & $\begin{array}{l}(0 \ldots 00211) \\
(0 \ldots 01201) \\
(0 \ldots 01210)\end{array}$ \\
\hline
\end{tabular}

3) $\operatorname{deg}=3$ для веса $(0,4,-3,-1)$;

случай $\mathfrak{s l}(5)$, избранные простые кокорни $(1,3)$ :

1) $\operatorname{deg}=0$ для веса $(-2,0,3,-3)$,

2) $\operatorname{deg}=1$ для веса $(-1,-3,4,0)$,

$3) \operatorname{deg}=1$ для веса $(3,-3,2,-2)$,

4) $\operatorname{deg}=1$ для веса $(4,-1,-1,-1)$;

случай $\mathfrak{s l}(4)$, избранные простые кокорни $(1,2)$ :

1) $\operatorname{deg}=1$ для веса $(-4,4,0)$,

2) $\operatorname{deg}=2$ для веса $(4,-1,-2)$,

3) $\operatorname{deg}=3$ для веса $(0,4,-4)$; 
ТАБлицА 3. Неголономные тензоры для флагов, ассоциированных с $\mathfrak{o}(2 n+1)$

\begin{tabular}{|c|c|c|c|c|}
\hline Вершина & $k(i)$ & $r k$ & Bec & $H^{1}$ \\
\hline \multirow[t]{3}{*}{1} & \multirow[t]{3}{*}{1} & 2 & $(3,1)$ & $(22)$ \\
\hline & & 3 & $(2,0,-2)$ & $(222)$ \\
\hline & & $3+k$ & $(2,0,-2, \ldots,-2)$ & $(2 \ldots 2)$ \\
\hline \multirow{4}{*}{2} & \multirow{4}{*}{2} & 3 & $\frac{k}{(0,1,-2)}$ & $(022)$ \\
\hline & & & $(-1,1,-1)$ & (121) \\
\hline & & $3+k$ & $(0,1,-2, \underbrace{-2, \ldots,-2})$ & $(02 \ldots 2)$ \\
\hline & & & $(-1,1,-1,-2, \ldots,-2)$ & $(1210 \ldots 0)$ \\
\hline \multirow{3}{*}{3} & \multirow{3}{*}{2} & $4+k$ & $\frac{k}{(-1,-2,0,1,-2, \ldots,-2)}$ & $(002 \ldots 2)$ \\
\hline & & & $(-1,0,1,-2-2, \ldots,-2)$ & $(01210 \ldots 0)$ \\
\hline & & & $k$ & \\
\hline$\ldots$ & $\ldots$ & $\ldots$ & $\ldots$ & $\ldots$ \\
\hline предпос- & \multirow[t]{3}{*}{2} & $5+k$ & $(-1, \underbrace{-2, \ldots,-2}-2,0,-1,-2)$ & $(0 \ldots 022)$ \\
\hline \multirow[t]{2}{*}{ ледний } & & & $(-1,-2, \ldots,-2,-1,0,-2,-2)$ & $(0 \ldots 0121)$ \\
\hline & & & $k$ & \\
\hline \multirow[t]{3}{*}{ последний } & \multirow[t]{3}{*}{1} & 2 & $(0,3)$ & - \\
\hline & & 3 & $(-1,0,3)$ & (123) \\
\hline & & $3+k$ & $(-1, \underbrace{-2, \ldots,-2}_{k},-1,1)$ & $(0 \ldots 0123)$ \\
\hline
\end{tabular}

ТАБлицА 4. Неголономные тензоры для флагов, ассоциированных с $\mathfrak{s p}(2 n)$

\begin{tabular}{|c|c|c|c|c|}
\hline Вершина & $k(i)$ & $r k$ & вес & $H^{1}$ \\
\hline \multirow[t]{3}{*}{1} & \multirow[t]{3}{*}{2} & 2 & $(3,0)$ & - \\
\hline & & 3 & $(2,1,-1)$ & - \\
\hline & & $3+k$ & $(2,-1, \underbrace{-2, \ldots,-2}_{k},-1)$ & - \\
\hline \multirow[t]{2}{*}{2} & \multirow[t]{2}{*}{2} & 3 & $\begin{array}{l}(1,2,-1) \\
(-2,1,0)\end{array}$ & (121) \\
\hline & & $4+k$ & $\begin{array}{l}(1,2,-2, \underbrace{-2, \ldots,-2}_{k},-1) \\
(-2,0,-1, \underbrace{-2, \ldots,-2}_{k},-1)\end{array}$ & $\begin{array}{l}(1210 \ldots 0) \\
(12 \ldots 21)\end{array}$ \\
\hline$\ldots$ & $\ldots$ & $\ldots$ & $\ldots$ & $\ldots$ \\
\hline пред-пред- & 2 & $4+k$ & $(\underbrace{-2, \ldots,-2},-2,0,1,-1)$ & $(0 \ldots 01210)$ \\
\hline последний & & & 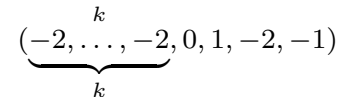 & $(0 \ldots 01211)$ \\
\hline $\begin{array}{l}\text { предпос- } \\
\text { ледний }\end{array}$ & 2 & $3+k$ & $\begin{array}{c}(\underbrace{-2, \ldots,-2}_{k}-2,1,0) \\
(\underbrace{-2, \ldots,-2}_{k},-1,0,-1)\end{array}$ & $(0 \ldots 0121)$ \\
\hline последний & 1 & 2 & $(1,3)$ & $(22)$ \\
\hline & & $3+k$ & $(\underbrace{-2, \ldots,-2}_{k},-2,-1,1)$ & $(0 \ldots 022)$ \\
\hline
\end{tabular}


случай $\mathfrak{s l}(4)$, избранные простые кокорни $(1, n)$ :

\begin{tabular}{|c|c|c|c|}
\hline степень & вектор: $n>4$ & вес: $n>4$ & вес: $n=4$ \\
\hline 1 & $\left(e_{2}^{n+1}\right)^{*} d e_{1}^{2} d e_{1}^{3}$ & $(4,-1,-1,0, \ldots, 0,-1)$ & $(4,-1,-2)$ \\
\hline \multirow{2}{*}{1} & симметричный & симметричный & симметричный \\
& приведенному выше & приведенному & приведенному \\
& относительно & выше & выше \\
& $n+1 \longleftrightarrow 1, n \longleftrightarrow 2$, и т.д. & & \\
\hline 2 & $\left(e_{2}^{n}\right)^{*} d e_{1}^{2} d e_{n}^{n+1}$ & $(3,-2,0, \ldots, 0,-2,3)$ & $(3,-4,3)$ \\
\hline
\end{tabular}

случай $\mathfrak{s l}(3)$ :

1) $\operatorname{deg}=\overline{4:}\left(e_{3}^{2}\right)^{*} d\left(e_{2}^{3}\right) d\left(e_{1}^{3}\right)$ для веса $(-1,5)$,

2) симметричный приведенному выше.

Модуль $\underline{H^{1}}$ : все векторы за исключением пункта 3 существуют при $2 \leqslant i \leqslant n-1$ :

1) $\operatorname{deg}=1:\left((i-1) e_{1}^{1}-e_{2}^{2}-\cdots-e_{i}^{i}\right)^{*} d e_{i}^{i+1}$ для веса $(\ldots, 0,-1,2,-1, .0, \ldots)$,

2) $\operatorname{deg}=1:\left((i-1) e_{1}^{1}-e_{2}^{2}-\cdots-e_{i}^{i}\right)^{*} d e_{1}^{2}$ для веса $(2,-1,0, \ldots)$,

3) $\operatorname{deg}=2:\left(e_{i}^{i+1}\right)^{*} d e_{i-1}^{i+2}-\left(e_{i-1}^{i+1}\right)^{*} d e_{i}^{i+2}-\left(e_{i}^{i+2}\right)^{*} d e_{i-1}^{i+1}+\left(e_{i-1}^{i+2}\right)^{*} d e_{i}^{i+1}$ для веса $(\ldots, 0,-1,0,2,0,-1,0, \ldots)$ (при $i=n-1$ для веса $(\ldots, 0,-1,0,2,0))$, существует при $3 \leqslant i \leqslant n-1$,

4) $\operatorname{deg}=2:\left(e_{i}^{i+1}\right)^{*} d e_{i}^{i+1}$ для веса $(\ldots, 0,-2,4,-2,0, \ldots)$,

$5) \operatorname{deg}=2$ : для веса $(1,0, \ldots, 0,1,-1,0, \ldots)$ :

$$
\begin{aligned}
& (n+1)\left((i-1) e_{1}^{1}-e_{2}^{2}-\cdots-e_{i}^{i}\right)^{*} d e_{1}^{i+1}- \\
& \quad-(i-1)(n-i+1) \sum_{2 \leqslant j \leqslant i}\left(\left(e_{j}^{i+1}\right)^{*} d e_{1}^{j}\right)+(n+2-i) \sum_{2 \leqslant j \leqslant i}\left(\left(e_{1}^{j}\right)^{*} d e_{j}^{i+1}\right),
\end{aligned}
$$

6) $\operatorname{deg}=2:\left(e_{1}^{2}\right)^{*} d e_{1}^{2}$ для веса $(4,-2,0, \ldots)$.

Б. Случай $\mathfrak{s p}(2 n)$ :

\begin{tabular}{|c|c|c|c|}
\hline степень & вес: $n>3$ & вес: $n=3$ & $H^{1}$ \\
\hline-1 & $(-2,0, \ldots, 0,-1,-2,3)$ & $(-3,-2,3)$ & - \\
\hline 1 & $(4,-3,0, \ldots, 0,-2,2)$ & $(4,-5,2)$ & $(0, \ldots, 0,-2,2)$ \\
1 & $(5,-2,-1,0, \ldots, 0)$ & $(5,-2,-1)$ & $(2,-1,0, \ldots, 0)$ \\
\hline
\end{tabular}

Существуют также неприводимые компоненты модуля $H^{1}$ степени 2 , их веса равны

$$
(0, \ldots, 0,-2,0,2), \quad(0, \ldots, 0,-4,4), \quad(1,0, \ldots, 0,-1,1), \quad(4,-2,0, \ldots, 0) .
$$

Исключительный случай $\mathfrak{s p}(4)$ :

$\underline{H^{2}}: \operatorname{deg}=3$ для веса $(6,-3)$ и $\operatorname{deg}=4$ для веса $(-4,5)$;

$\underline{H^{1}}: \operatorname{deg}=1$ для весов $(-2,2)$ и $(2,-1) ; \operatorname{deg}=2$ для весов $(-4,4),(0,1)$ и $(4,-2)$.

\section{8. ЯВНЫЕ РЕЗУЛЬТАТЫ: $G(2)$-СТРУКТУРЫ}

8.1. $G(2)$-структуры. Пусть $M$ - многообразие с заданной $G=G(2)$-структурой, т.е. касательное пространство $T_{m} M$ в каждой точке $m$ является $G(2)$-модулем, 
изоморфным первому фундаментальному модулю (ср. [45]). Мы хотели бы знать, в какой мере "положительная 3-форма" (инвариант $G(2)$-структуры, аналогичный метрике в римановом случае, т.е. в случае $\mathfrak{o}(n)$-структуры), см. [45], может быть приведена к каноническому виду. Ниже приводится описание пространства препятствий к каноничности. Поскольку $\mathfrak{g}(2) \subset \mathfrak{o}(7)$ и $\mathfrak{o}(n)_{1}=0$ при $n>2$, легко заметить, что $\left(\mathfrak{g}_{-1}, \mathfrak{g}_{0}\right)_{*}=\mathfrak{g}_{-1} \oplus \mathfrak{g}_{0}$.

ТЕОРемА. Как $\mathfrak{g}_{0}$-модуль, пространство $H^{2}\left(\mathfrak{g}_{-1} ;\left(\mathfrak{g}_{-1}, \mathfrak{g}_{0}\right)_{*}\right)$ является прямой суммой неприводимых $G(2)$-модулей, для которых старшие веса и порядки соответствующих структурных функций приведены в следующей таблице:

\begin{tabular}{|c|c|}
\hline вес & порядок \\
\hline$(0,0),(0,1),(1,0),(2,0)$ & 1 \\
\hline$(0,2)$ & 2 \\
\hline
\end{tabular}

Равенство нулю структурных функций первого порядка (соответствующие уравнения см. в [45]) является аналогом связей Весса-Зумино в супергравитации [36].

Из списка простых $\mathbb{Z}$-градуированных алгебр Ли мы выводим, что не существует аналога классической области с конформной $G=C G(2)$-структурой, даже неголономной. Напротив, среди простых супералгебр Ли существует ровно одна исключительная супералгебра, обладающая нужной градуировкой. Вычислим соответствующее пространство структурных функций.

\section{2. $C G(2)$-структура на пространстве $\mathbb{C} P^{1,7}$ с неголономным распреде-} лением. Рассмотрим пространство $\mathbb{C} P^{1,7}$ как фактор простой супергруппы $A G(2)$ по параболической подгруппе $P$, для которой супералгебра Ли р получается следующим образом. Рассмотрим супералгебру Ли $\mathfrak{a g}(2)=\operatorname{Lie}(A G(2))$ супергруппы $A G(2)$, построенной по матрице Картана $\left(\begin{array}{rrr}0 & 1 & 0 \\ -1 & 2 & -3 \\ 0 & -1 & 2\end{array}\right)$ [65]. Тогда подалгебра $\mathfrak{p}$ натягивается на элементы неположительной степени в градуировке $(1,0,0)$ супералгебры $\mathfrak{a g}(2)$.

В этой градуировке $\mathfrak{g}_{0}=\mathfrak{g}(2) \oplus \mathfrak{z}$ с одномерным центром $\mathfrak{z}$ положим $\widehat{\mathfrak{g}_{0}}=\mathfrak{g}(2)$. Теперь уже не так легко, как в п. 8.1, увидеть, что $\left(\mathfrak{g}_{-}, \mathfrak{g}_{0}\right)_{*}=\mathfrak{a g}(2)$ и $\left(\mathfrak{g}_{-}, \widehat{\mathfrak{g}_{0}}\right)_{*}=$ $\mathfrak{g}_{-} \oplus \widehat{\mathfrak{g}_{0}}$, тем не менее эти равенства верны. Поскольку никакой суперверсии теоремы ББВ не существует (ср. [50], [66]), мы использовали для вычислений пакет SuperLie и получили следующее утверждение.

УТВЕРЖДЕНИЕ. Как $\mathfrak{g}_{0}=\mathfrak{s l}(2) \oplus \mathfrak{g}(2)$-модуль, пространство $H^{2}\left(\mathfrak{g}_{-1} ; \mathfrak{a g}(2)\right)$ неголономных конформных структурных функиий является неприводимым модулем со стариим весом $(5,0,1)$.

Как $\widehat{\mathfrak{g}_{0}}=\mathfrak{z} \oplus \mathfrak{g}(2)$-модуль, пространство $H^{2}\left(\mathfrak{g}_{-1} ;\left(\mathfrak{g}_{-}, \widehat{\mathfrak{g}_{0}}\right)_{*}\right)$ неголономных римановых структурных функиий является прямой суммой неприводимых модулей со следующими старшими весами: $(5,0,1),(6,1,0),(7,0,1)$.

ЗАмЕчАНИЕ. В попытке понять смысл тензоров $V\left(\mathfrak{g}_{i}\right)$ из [45], где появляются внешние степени пространства $\mathfrak{g}_{-1}$, мы предположили, что эти тензоры $V\left(\mathfrak{g}_{i}\right)$ могут быть связаны с $G(2)$-структурой на чисто нечетном суперпространстве. Однако, 
вычислив соответствующие структурные функции, веса и порядки которых приведены в следующей таблице:

\begin{tabular}{|c|c|}
\hline вес & порядок \\
\hline$(0,1),(1,0),(3,0)$ & 1 \\
\hline$(0,1)$ & 2 \\
\hline
\end{tabular}

мы увидели, что они не совпадают с $V\left(\mathfrak{g}_{i}\right)$, так что смысл тензоров $V\left(\mathfrak{g}_{i}\right)$ все еще остается для нас загадкой.

Благодарности. Д. Лейтес благодарен А. Премету, И. Щепочкиной, И. Бернштейну за щедрую помощь; IHES, Försäkringskassan и MPIMiS за финансовую поддержку, а также рецензентам за ценные комментарии. В заключительной стадии работа была поддержана РФФИ (грант № 06-01-00469).

\section{Список литературы}

[1] Г. Герц, Принципы механики, изложенные в новой свлзи, АН СССР, М., 1959.

[2] А. Пуанкаре, "Идеи Герца в механике", в кн.: Г. Герц, Приниипь механики, изложеннъе в новой связи, АН СССР, М., 1959, 310-333.

[3] А.М. Вершик, В.Я. Гершкович, "Неголономные динамические системы. Геометрия распределений и вариационные задачи", Итоги науки и техники. Современные проблемы математики. Фундаментальные направления, 16, ВИНИТИ, М., 1987, 5-85.

[4] А.М. Вершик, "Классические и неклассические динамики со связями", Геометрия u топология в глобальных нелинейных задачах, Новое в глобальном анализе, ВГУ, Воронеж, 1984, 23-48.

[5] A. Agrachev, Yu. Sachkov, Control Theory from the Geometric Viewpoint, Encyclopaedia Math. Sci., 87, Springer, Berlin, 2004.

[6] A. M. Bloch, Nonholonomic Mechanics and Control, Interdiscip. Appl. Math., 24, Springer, New York, 2003.

[7] В. М. Сергеев, Предель рачиональности. Термодинамический подход к теории экономического равновесия, Фазис, М., 1999.

[8] В. В. Козлов, Тепловое равновесие по Гиббсу и Пуанкаре, Соврем. матем., Ин-т компьютерных исследований, Москва-Ижевск, 2002.

[9] V. Sergeev, The Thermodynamical Approach to Market, MPIMiS preprint 76/2006 (translated from the Russian and edited by Dimitry Leites), http://www.mis.mpg.de/preprints/ 2006/prepr2006_76.html.

[10] D. Leites, Homology Homotopy Appl., 4:2(2) (2002), 397-407; math.RT/0202213; Proc. Workshop on mathematical physics and geometry (ICTP, Trieste, Italy, March 4-15, 1991), http://agenda.ictp.trieste.it/agenda/current/fullAgenda.php?ida=a02210.

[11] D. Leites, E. Poletaeva, "Supergravities and contact type structures on supermanifolds", Second International Conference on Algebra (Barnaul, Russia, 1991), Contemp. Math., 184, AMS, Providence, RI, 1995, 267-274.

[12] P. Grozman, D. Leites, "From supergravity to ballbearings", Supersymmetries and Quantum Symmetries (Dubna, 1997), Lecture Notes in Phys., 524, eds. J. Wess, E. $\dot{A}$. Ivanov, Springer, Berlin, 1999, 58-67.

[13] N. Tanaka, J. Math. Soc. Japan, 22 (1970), 180-212.

[14] N. Tanaka, J. Math. Kyoto Univ., 10 (1970), 1-82.

[15] N. Tanaka, Hokkaido Math. J., 8:1 (1979), 23-84. 
[16] K. Yamaguchi, "Differential systems associated with simple graded Lie algebras", Progress in Differential Geometry, Adv. Stud. Pure Math., 22, Math. Soc. Japan, Tokyo, 1993, 413-494.

[17] K. Yamaguchi, T. Yatsui, "Geometry of higher order differential equations of finite type associated with symmetric spaces", Lie groups, geometric structures and differential equations - one hundred years after Sophus Lie (Kyoto/Nara, 1999), Adv. Stud. Pure Math., 37, eds. T. Morimoto, H. Sato, K. Yamaguchi, Math. Soc. Japan, Tokyo, 2002, 397-458.

[18] V. Dragovic, B. Gajic, Regul. Chaotic Dyn., 8:1 (2003), 105-123; math-ph/0304018.

[19] P. Grozman, SuperLie, http://www .equaonline.com/math/SuperLie.

[20] R. J. Baston, Duke Math. J., 63:1 (1991), 81-112; 113-138.

[21] A. Čap, H. Schichl, Hokkaido Math. J., 29:3 (2000), 453-505.

[22] A. Čap, J. Reine Angew. Math., 2005, № 582, 143-172; math.DG/0102097.

[23] A. Čap, J. Slovák, Math. Scand., 93:1 (2003), 53-90; math.DG/0001166.

[24] A. Čap, J. Slovák, V. Souček, Ann. of Math. (2), 154:1 (2001), 97-113.

[25] A. Čap, J. Slovák, Rend. Circ. Mat. Palermo (2) Suppl., 1996, № 43, 95-101.

[26] A. S. Galperin, E. A. Ivanov, V. I. Ogievetsky, E. S. Sokatchev, Harmonic Superspace, Cambridge Monogr. Math. Phys., Cambridge Univ. Press, Cambridge, 2001.

[27] P. Heslop, P. S. Howe, Class. Quantum Grav., 17:18 (2000), 3743-3768.

[28] K. Ehlers, J. Koiller, R. Montgomery, P. Rios, "Nonholonomic systems via moving frames: Cartan equivalence and Chaplygin Hamiltonization", The Breadth of Symplectic and Poisson Geometry, Progr. Math., 232, eds. J. E. Marsden, T. S. Ratiu, Birkhäuser, Boston, MA, 2005, 75-120.

[29] J. N. Tavares, J. Geom. Phys., 45:1-2 (2003), 1-23.

[30] И. М. Щепочкина, ТМФ, 147:3 (2006), 450-469; math.RT/0509472.

[31] S. Vacaru, Interactions, Strings and Isotopies in Higher Order Anisotropic Superspaces, Hadronic Press Monogr. Math., Hadronic Press, Palm Harbor, FL, 1998.

[32] А. Гончаров, Функи. анализ и его прилож., 15:3 (1981), 83-84; Selecta Math. Soviet., 6:4 (1987), 307-340.

[33] B. Kostant, "A generalization of the Bott-Borel-Weil theorem and Euler number multiplets of representations", Conférence Moshé Flato, 1999. Vol. 1. Quantization, Deformations, and Symmetries (Dijon, France, 1999), Math. Phys. Stud., 21, Kluwer, Dordrecht, 2000, 309-325; Lett. Math. Phys., 52:1 (2000), 61-78.

[34] S. Sternberg, Lectures on Differential Geometry, 2nd ed., Chelsey Publ., New York, 1983.

[35] V. Guillemin, Trans. Amer. Math. Soc., 116 (1965), 544-560.

[36] Ю. Весс, Дж. Беггер, Суперсимметрия и супергравитация, УРСС, М., 1998.

[37] Ю. И. Манин, Калибровочные поля и комплексная геометрия, Наука, М., 1984.

[38] D. Leites, I. Shchepochkina, Classification of the simple Lie superalgebras of vector fields, preprint MPIM-2003-28.

[39] I. Shchepochkina, C. R. Acad. Bulgare Sci., 36:3 (1983), 313-314.

[40] И. М Щепочкина, Функи. анализ и его прилож., 33:3 (1999), 59-72.

[41] I. Shchepochkina, Represent. Theory, 3:13 (1999), 373-415.

[42] P. Woit, Quantum field theory and representation theory: a sketch, hep-th/0206135.

[43] D. Leites, E. Poletaeva, V. Serganova, J. Nonlinear Math. Phys., 9:4 (2002), 394-425; math.DG/0306209.

[44] M. Atiyah, E. Witten, Adv. Theor. Math. Phys., 6:1 (2002), 1-106.

[45] R. L. Bryant, Some remarks on $G_{2}$-structures, math.DG/0305124.

[46] V. V. Fock, A. B. Goncharov, "Cluster X-varieties, amalgamation and Poisson-Lie groups", Algebraic Geometry and Number Theory, Progr. Math., 253, Birkhäuser, Boston, MA, 2006, 27-68; math.RT/0508408.

[47] P. Grozman, D. Leites, SuperLie and problems (to be) solved with it, preprint MPIM-Bonn 2003-39. 
[48] E. Poletaeva, Analogs of the Riemannian and Penrose tensors on supermanifolds, math.RT/0510165.

[49] D. Leites, V. Serganova, G. Vinel, "Classical superspaces and related structures", Differential geometric methods in theoretical physics, Proc. 19th Int. Conf. (Rapallo, Italy, 1990), Springer Lect. Notes Phys., 375, eds. U. Bruzzo et.al., 1991, 286-297.

[50] I. Penkov, V. Serganova, Ann. Inst. Fourier (Grenoble), 39:4 (1989), 845-873.

[51] Y. Se-Ashi, Hokkaido Math. J., 17:2 (1988), 151-195.

[52] T. Morimoto, Hokkaido Math. J., 22:3 (1993), 263-347; "Lie algebras, geometric structures and differential equations on filtered manifolds", Lie Groups, Geometric Structures and Differential Equations - One Hundred Years After Sophus Lie (Kyoto/Nara, 1999), Adv. Stud. Pure Math., 37, eds. T. Morimoto, H. Sato, K. Yamaguchi, Math. Soc. Japan, Tokyo, 2002, 205-252.

[53] Б. Л. Фейгин, Д.Б. Фукс, “Когомологии групп и алгебр Ли”, Группь Ли и алгебpы Ли, 2, Итоги науки и техники. Современные проблемы математики. Фундаментальные направления, 21, ВИНИТИ, М., 1988, 121-209.

[54] Ш. Кобаяси, К. Номидзу, Основы дифференциальной геометрии, т. 1, 2, Наука, М., 1981.

[55] V. Gershkovich, A. Vershik, J. Geom. Phys., 5:3 (1988), 407-452; А. М. Вершик, В. Я. Гершкович, Записки науч. сем. ЛОМИ, 172 (1989), 21-40.

[56] R. Montgomery, "Engel deformations and contact structures", Northern California Symplectic Geometry Seminar, Amer. Math. Soc. Transl. Ser. 2, 196, AMS, Providence, RI, 1999, 103-117.

[57] V. Ovsienko, S. Tabachnikov, Projective differential geometry old and new. From Schwarzian derivative to cohomology of diffeomorphism groups, Cambridge Tracts in Math., 165, Cambridge Univ. Press, Cambridge, 2005; http://www.math.psu.edu/ tabachni/prints/preprints.html.

[58] I. Biswas, A. Raina, Internat. Math. Res. Notices, 1996, № 15, 753-768; 1999, № 13, 685-716; Differential Geom. Appl., 15:3 (2001), 203-219.

[59] П. Грозман, Д. Лейтес, И. Щепочкина, "The analogs of the Riemann tensor for exceptional structures on supermanifolds", Фундаментальная математика сегодня. К десятилетию Независимого Московского Университета (Москва, 2001), ред. С. К. Ландо, О. К. Шейнман, МЦНМО, М., 2003, 89-109; preprint MPIM-2003-18; math.RT/0509525.

[60] M. Zhitomirskii, Typical Singularities of Differential 1-forms and Pfaffian Equations, Transl. Math. Monogr., 113, AMS, Providence, RI; Mir Publ., Moscow, 1992.

[61] A. Lebedev, D. Leites, I. Shereshevskii, "Lie superalgebra structures in $C^{\bullet}(\mathfrak{n} ; \mathfrak{n})$ and $H^{\bullet}(\mathfrak{n}, \mathfrak{n}) "$, Lie Groups and Invariant Theory, Amer. Math. Soc. Transl. Ser. 2, 213, ed. É. Vinberg, AMS, Providence, RI, 2005, 157-172; math.KT/0404139.

[62] I. N. Bernstein, I. M. Gelfand, S. I. Gelfand, "Differential operators on the base affine space and a study of g-modules", Lie Groups and Their Representations (Budapest, 1971), Halsted, New York, 1975, 21-64.

[63] Н. Бурбаки, Группы и алгебры Ли, Гл. IV-VI, Мир, М., 1972.

[64] Э. Б. Винберг, А. Л. Онищик, Семинар по группам Ли и алгебраическим группам, Наука, М., 1988.

[65] P. Grozman, D. Leites, Czechoslovak J. Phys., 51:1 (2001), 1-21; hep-th/9702073.

[66] И. Б. Пенков, “Теория Бореля-Вейля-Ботта для классических супергрупп Ли”, Итоги науки и техники. Современные проблемы математики. Новейшие достижения, $\mathbf{3 2}$, ВИНИТИ, М., 1988, 71-124.

Поступила в редакцию 6.07.2006, после доработки 30.12.2006 